\title{
Renormalization and tensor product states in spin chains and lattices
}

\author{
J. Ignacio Cirac \\ Max-Planck-Institut für Quantenoptik, Hans-Kopfermann-Str. 1, D-85748 Garching, \\ Germany. \\ E-mail: ignacio.cirac@mpq.mpg.de \\ Frank Verstraete \\ Fakultät für Physik, Universität Wien, Boltzmanngasse 5, A-1090 Wien, Austria. \\ E-mail: frank.verstraete@univie.ac.at
}

\begin{abstract}
We review different descriptions of many-body quantum systems in terms of tensor product states. We introduce several families of such states in terms of known renormalization procedures, and show that they naturally arise in that context. We concentrate on Matrix Product States, Tree Tensor States, Multiscale Entanglement Renormalization Ansatz, and Projected Entangled Pair States. We highlight some of their properties, and show how they can be used to describe a variety of systems.

PACS numbers: 02.70.-c,03.67.-a,05.30.-d,03.65.Ud
\end{abstract}




\section{Introduction}

Many-body quantum states appear in many contexts in Physics and other areas of Science. They are very hard to describe, even computationally, due to the number of parameters required to express them, which typically grows exponentially with the number of particles. Let us consider a spin chain of $N$ spin $s$ systems. If we write an arbitrary state in the basis $\left|n_{1}, \ldots, n_{N}\right\rangle$, where $n_{k}=1, \ldots,(2 s+1)$, we will have to specify $(2 s+1)^{N}$ coefficients. Even if $s=1 / 2$, and $N \sim 50$, it is impossible to store such a number of coefficients in a computer. Furthermore, even if that would be possible, whenever we want to make any prediction, like the expectation value of an observable, we will have to operate with those coefficients, and thus the number of operations (and therefore the computational time) will inevitably grow exponentially with $N$.

In many important situations, one can circumvent this problem by using certain approximations. For example, sometimes it is possible to describe the state in the socalled mean field approximation, where we write $|\Psi\rangle=\left|\phi_{1}, \ldots, \phi_{N}\right\rangle$, ie. as a product state. Here, we just have to specify each of the $\left|\phi_{M}\right\rangle$, and thus we need only $(2 s+1) N$ coefficients. This method and its extensions, even though it has a restricted validity in general, has been very successfully used to describe many of the phenomena that appear in quantum many-body systems. This indicates that, among all possible states, the ones that are relevant for many practical situations possess the same properties as product states. Another very successful method is renormalization [1, 2] which, in the context of spin chains and lattices, tries to obtain the physics of the low energy states by grouping degrees of freedom and defining new ones that are simple to handle, so that at the end we can cope with very large systems and using very few parameters. There exist very successful methods to uncover the physical properties of many-body systems which exploit numerical approaches. The first one is Quantum Monte Carlo, which samples product states in order to get the expectation values of physical observables. Another one is Density Matrix Renormalization Group (DMRG) [3, 4], which is specially suited for $1 \mathrm{D}$ lattices, and is based on renormalization group ideas.

\subsection{DMRG and Tensor Product States}

Wilson's renormalization method provided a practical way of qualitatively determining the low energy behavior of some of those systems. However, it was by no means sufficient to describe them quantitatively. In 1991, Steve White [3, 4] proposed a new way of performing the renormalization procedure in 1D systems, which gave extraordinary precise results. He developed the DMRG algorithm, in which the renormalization procedure takes explicitly into account the whole system at each step. This is done by keeping the states of subsystems which are relevant to describe the whole wavefunction, and not those that minimize the energy on that subsystem. The algorithm was rapidly extended and adapted to different situations [5, 6], becoming the method of choice for 1D systems. In 1995, Ostlund and Rommer [7] realized that the state resulting from the DMRG algorithm could be written as a so-called Matrix Product State (MPS), ie, in 
terms of products of certain matrices (see also [8, 9, 10]). They proposed to use this set of states as a variational family for infinite homogeneous systems, where one could state the problem without the language of DMRG, although the results did not look as precise as with the finite version of that method. Those states had appeared in the literature in many different contexts and with different names. First, as a variational Ansatz for the transfer matrix in the estimation of the partition function of a classical model [11. Later on, in the AKLT model in 1D [12, 13], where the ground state has the form of a valence bond solid (VBS) which can be exactly written as a MPS. Translationally invariant MPS in infinite chains were thoroughly studied and characterized mathematically in full generality in Ref. [14], where they called such family finitely correlated states (FCS). The name MPS was coined later on by Klümper et al [15, 16], who introduced different models extending the AKLT where the ground state had the explicit FCS form. All those studies were carried out for translationally invariant systems, where the matrices associated to each spin do not depend on the position of the spin. An extension of the work of Fannes et al [14] to general MPS (ie finite and non-homogeneous states) appeared much later on [17].

Given the success of DMRG in 1D, several authors tried to extend it to higher dimensions. The first attempts considered a $2 \mathrm{D}$ system as a chain, and used DMRG directly on the chain [18, 19, 20], obtaining much less precise results than in 1D. Another attempt considered homogeneous 3D classical system and used ideas taken from DMRG to estimate the partition function of the Ising Model [21]. A different approach was first suggested by Sierra and Martin-Delgado [22] inspired by the ideas of Ostlund and Rommer [7]. They introduced two families of translationally invariant states, the vertexand face-matrix product state Ansätze, and proposed to use them variationally for 2D systems, in the same way as Ostlund and Rommer used FCS in 1D. The first family generalized the AKLT 2D VBS state [12] in as much the same way as FCS did it in 1D. The second one was inspired by interaction-round-the-face models in (classical) Statistical Mechanics. The inclusion of few parameters in the VBS wavefunction of AKLT to extend that model was first suggested in Ref. [23]. The authors also showed that the calculation of expectation values in those VBS could be thought of as evaluating a classical partition function, something they did using Monte Carlo methods [23], and Hieida et al using ideas taken from DMRG [24]. Later on, Nishino and collaborators used the representations proposed by Sierra and Martin-Delgado, as well as another one they called interaction-round-a-face (inspired by the specific structure of the transfer matrix of the classical Ising model) to determine the partition function of the classical Ising Model in 3D variationally [25, 26, 27, 28, 29]. For instance, in [28] a vertical density matrix algorithm was introduced to calculate thermodynamical properties of that model based on the the interaction-round-a-face representation and in [27] a perturbation approach was taken for the vertex matrix product state, which turn out to be numerically unstable. Eventually, quantum systems at zero temperature were considered by direct minimization of trial wavefunctions of the VBS type with few variational parameters [30, 31]. In summary, most of the attempts in 2D quantum systems (and 3D classical 
ones) tried to generalize the method of Ostlund and Rommer [7] by using families of states that extended FCS to higher dimensions, and dealing with infinite homogenous systems. FCS and their extensions were based on tensors contracted in some special ways, and thus all of them were called tensor product states (TPS). Nevertheless, no DMRG-like algorithm for 2D or higher dimensions was put forward (except for the direct application of DMRG by considering the 2D system as a $1 \mathrm{D}$ chain).

The success of DMRG for 1D systems indicated that the family of states on which it based, namely MPS, may provide an efficient and accurate description of spin chain systems. In higher dimensions, however, the situation was much less clear since only infinite systems were considered and the numerical results were not entirely satisfactory.

\subsection{The corner of Hilbert space}

One can look at the problem of describing many-body quantum systems from a different perspective. The fact that product states in some occasions may capture the physics of a many-body problem may look very surprising at first sight: if we choose a random state in the Hilbert space (say, according to the Haar measure) the overlap with a product state will be exponentially small with $N$. This apparent contradiction is resolved by the fact that the states that appear in Nature are not random states, but they have very peculiar forms. This is so because of the following reason. If we consider states in thermal equilibrium, each state of a system, described by the density operator $\rho$, is completely characterized by the Hamiltonian describing that system, $H$, and the temperature, $T, \rho \propto e^{-H / T}$. In all systems we know, the Hamiltonian contains terms with at most $k$-body interactions, where $k$ is a fixed number independent of $N$ which typically equals 2 . We can thus parameterize all possible Hamiltonians in Nature in terms of $(N, k) \times(2 s+1)^{2 k}$. The first term is the number of groups of $k$ spins, whereas the second one gives the number of parameters of a general Hamiltonian acting on $k$ spins. This number scales only polynomially with $N$, and thus all possible density operators will also depend on a polynomial number of parameters. In practice, if we just have 2-body interactions (ie, $\mathrm{k}=2$ ), and short-range interactions, the number will be linear in $N$. If we additionally have translational symmetry, the number will even be independent of $N$. This shows that even though we just need an exponential number of parameters to describe a general state, we need very few to describe the relevant states that appear in Nature. In this sense, the relevant states are contained in "a corner of the Hilbert space". This representation is, however, not satisfactory, since it does not allow one to calculate expectation values.

These facts define a new challenge in many-body quantum physics, namely, to find good and economic descriptions of that corner of Hilbert space. That is, a family of states depending on few parameters (which increase only polynomially with $N$ ), such that all relevant states in Nature can be approximated by members of such family. If we are able to do that, as well as to characterize and study the properties of such family, we would have a new language to describe many-body quantum systems which may 
be more appropriate than the one we use based on Hilbert space expansions. Apart from that, if we find algorithms which, for any given problem (say, a Hamiltonian and a temperature), allows us to determine the state in the family which approaches the exact one, we will have a very powerful numerical method to describe quantum many-body systems. It is clear that product states are not enough for that task, since they do not posses correlations (nor entanglement), something that is crucial in many physical phenomena. So, the question is how to extend product states in a way that they cover the relevant corner of Hilbert space.

One possible strategy to follow is to determine a property that all the states on that corner have, at least for a set of important problems. If we then find a family of states which includes all states with that particular property, we will have succeeded in our challenge. But, what property could that be? Here, the answer may come from ideas developed in the context, among others, of quantum information theory. One of such ideas appears for the subclass of problems with Hamiltonians that contain finite-range interactions (ie, two spins interact if they are at a distance smaller than some constant), have a gap (ie, for all $N, E \geq E_{0}+\Delta$, where $E$ is the energy of any excited state, $E_{0}$ that of the ground state, and $\Delta>0$ ), and are at zero temperature. In that case, the so-called area law naturally emerges [32, 33, 34, 35, 36, 37]. It states that for the ground state of such a Hamiltonian, $\left|\Psi_{0}\right\rangle$, if we consider a block, $A$, of neighboring spins, the von Neumann entropy of the reduced density operator of such a region, $\rho_{A}$, scales with the number of particles at the border of that region. This is quite remarkable, since the von Neumann entropy being an extensive quantity, for a random state it will scale with the number of particles in $A$, and not in the border. The area law has been proven in 1D spin chains [38], and it is fulfilled for all Hamiltonians we know in higher dimensions. Even for critical systems, where the gap condition is not fulfilled, only a slight violation occurs (namely that it is proportional to the number of spins at the border, $L$, times $\log L)$ [36, 39, 40]. What happens at finite temperature? In that case one can also find a global property of all states in the corner of Hilbert space which extends the area law. In contrast to the zero temperature case, now this can be rigorously proven for any Hamiltonian possessing finite-range interactions in arbitrary dimensions [41]. The property is the following: given a region $A$ as before, the mutual information between the spins in that region and the rest of the spins (in region $B$ ) is bounded by a constant times the number of spins at the border divided by the temperature. Here, the mutual information is defined as $I(A: B)=S\left(\rho_{A}\right)+S\left(\rho_{B}\right)-S\left(\rho_{A B}\right)$, where $S$ is the von Neumann entropy, and $\rho_{X}$ is the density operator corresponding to region $X$.

There is a way of constructing families of states explicitly fulfilling the area law. Let us first consider a 1D chain with two spins per site in which we entangle each of them with the nearest neighbor spins (to the right and to the left, respectively). If we now consider a block of neighboring sites, only the outermost spins with contribute to the entropy of that block, and thus the area law will be fulfilled. Furthermore, if we project in each site the state of the two spins onto a subspace of lower dimension, the resulting state will also fulfill the area law. This construction can be straightforwardly 
extended to higher dimensions, just by replacing each spin in the lattice by $z$ auxiliary spins which are pair-wise entangled with their neighbors (here $z$ is the coordination number of the lattice). By projecting the state of the auxiliary spins onto the Hilbert space of the original spin at each site, we obtain a state automatically fulfilling the area law. This construction, which is inspired by the VBS [12], was introduced in the context of quantum information in order to study localizable entanglement of spin chains [42] and to give an alternative explanation of the measurement based model of quantum computation [43]. Later on, it was extended to describe general spin lattice systems in [44, where the resulting states where called Projected Entangled-Pair States (PEPS), given the way they were defined. In that work, a method to approximate the ground state wavefunction in terms of PEPS for finite systems was introduced, which allowed to find the optimal projectors at each site in very much the same way as DMRG does it in 1D. Thus, the method can be considered as a truly extension of the DMRG algorithm to higher dimensions, although computationally it is much more demanding. The key ingredient in that method was a new algorithm that allows one to optimally approximate arbitrary states by PEPS in a variational fashion.

By explicitly expressing the projectors appearing in the PEPS in an orthonormal basis, one can immediately see that they have a tensor product form. In fact, in 1D the family of PEPS coincides with the MPS [42]. In higher dimensions, if one takes an infinite system and chooses all the projectors to be identical, the so-called iPEPS [45] coincide with the vertex matrix product ansatz introduced by Sierra and Martin-Delgado [22. The PEPS construction, however, apart from giving rise to (finite) DMRG-like algorithms in higher dimensions, gives a clear picture of the entanglement properties of those states (as, eg, related to the area law) as well as how correlations are carried over by the entangled auxiliary particles. More importantly, it can be used to extend the PEPS to fermionic systems still keeping all the properties that made them special [46].

The fact that PEPS fulfill the area law puts them in a privileged position to efficiently describe the corner of Hilbert space. In fact, in 1D systems at zero tempeature it is possible to show: (i) that all gapped Hamiltonians with finite-range interactions fulfill the area law [38]; (ii) all states fulfilling the area law (even with logarithmic corrections) can be efficiently approximated by MPS with a number of parameters that only scales polynomially with $N$ [49] (see also [50]). This means that for those systems at zero temperature we have found we were looking for and explains why the celebrated DMRG method introduced by White [3, 4] gives extraordinary approximations to the ground state of (finite) 1D spin chains.

A complementary approach to determine the interesting corner of Hilbert space has been pursued by Hastings [51] (see also [52]). Very remarkably, he has been able to prove that, for any finite temperature in any dimensions and Hamiltonian with finiterange interactions, PEPS efficiently describe the thermal state in the sense that they approximate it arbitrarily close with a polynomial number of parameters (Note that mixed state can be described in terms of PEPS using the techniques of purification [47, 48]). This shows that, for those problems, the corner of Hilbert space has been 
identified. Note that the restriction of finite temperature does not matter in many practical situations, since in any system we will always have that. It is, nevertheless, interesting to investigate what happens at exactly zero temperature. In that case, if one imposes a natural condition on the Hamiltonian (related with how the density of states above the ground sate grow), Hastings has also proven that PEPS provide an efficient description (note that for 1D systems the area law already implies that [49]). In summary, MPS in 1D and PEPS in higher dimensions provide us with accurate descriptions of the states that appear in Nature under the conditions specified above (most notably, short-range interactions).

\subsection{Tensor product states and renormalization group methods}

All the states above fall into a general class of states which can be called TPS $\ddagger$ This class also contains states like Tree Tensor States (TTS) [53] or Multi-scale Renormalization Ansatz (MERA) [54, 55]. It is characterized by the fact that the states are specified in terms of a few tensors, of the order of $N$, each of them having a small rank and low dimensions. Thus, they provide economic descriptions of certain quantum states. During the last years a number of algorithms have been proposed to determine those TPS for specific problems, giving rise to new numerical methods suitable to describe certain many-body quantum systems. The purpose of the present paper is to provide a pedagogical review of some of the most important TPS, and to connect them to the successful real-space renormalization procedures that have been used in Condensed Matter Physics for many years now. As we shall explain, most of the TPS lie at the basis of such procedures. Making the connection between those two problems (renormalization and tensor product states) offers a new perspective for both of them, and explains the success of some schemes from a different point of view. In particular, it relates the structure of the states that appear in Nature under some conditions with the ones that naturally appear in every renormalization procedure. We will mostly concentrate on MPS and PEPS, but we will also cover in part TTS and MERA, and briefly describe the rest. Note that, in the case of PEPS, we have not been able to connect them to any known renormalization scheme. Nevertheless, it may be interesting to find such a renormalization procedure since it may give another insight in the field of Condensed Matter Physics. Furthermore, very recently, several renormalization methods have been introduced in order to determine expectation values of this and other kinds of TPS [56, [57, 58], which is another evidence of the strong bonds existing between these two fields.

This paper is organized as follows. Section II deals with MPS. We first introduce them in terms of a renormalization procedure, and then explain two different methods to carry out that procedure: real-space renormalization [1] and density matrix renormalization group (DMRG) [3]. We also present them from a quantum information $\ddagger$ Several authors have recently started calling this family of states tensor network states (TNS). Here, for historical reasons, we will call them TPS. 
perspective, as the states that can be obtained by projecting pairs of entangled states into lower dimensional subspaces. This provides a physical picture of the states, which can be easily extended to higher dimensions and fermionic systems. We also review how expectation values can be efficiently determined, and introduce a graphical representation of the state which strongly simplifies the notation and which will be used later on instead of complex formulas. We show how MPS can be generated by a sequential application of quantum gates [59] which, apart from providing us with a specific recipe of how to engineer those states in practice, will allow us to connect MPS and TTS in a simple way. Then we illustrate how to approximate arbitrary states by MPS. This provides the basis of several algorithms that have been recently introduced to determine the ground state in different situations [10, 61, 60], time evolution [62, 63, 64, 65, 66, 47, 67, 68, 69], and thermal states [47, 48, 70] of 1D spin chains. Finally, we introduce matrix product operators [47, 48], and show how they can be used to determine expectation values in a different way or to describe thermal states via the purification procedure. Section III contains a summary of a different renormalization method from which TTS naturally emerge. As in the case of MPS, we show how one can determine expectation values, and other quantities. In fact, it will become apparent that the algorithms described in the previous section can be extended to TTS without much effort. We also relate MPS and TTS showing that one can be expressed in terms of the other one with a logarithmic effort. Then, in Section IV we review yet another renormalization procedure [84, 85] which allows us to introduce the MERA [54, 55] from a different perspective. As opposed to the TTS, MERA can be extended to higher dimensions still fulfilling the area law, which makes them suitable to study spin systems beyond chains. One way of generalizing MPS to higher dimensions is through the PEPS, which are the subject of Section V. As opposed to the previous sections, PEPS are not introduced in terms of a renormalization group procedure, but following the intuition provided by the area law and the entanglement present in the state. We finish the paper by reviewing some other extensions of MPS to higher dimensions (for homogeneous and infinite systems).

Although we briefly mention how to build algorithms using TPS, this is not the main purpose of this paper. For the reader interested in the algorithms, rather than in the way they appear and some of their properties, we recommend to have a look at Ref. [71]. For the reader interested in the mathematical properties of MPS and PEPS, as well as the development of a whole theory of many-body states based on that representation, we refer to the paper [72].

In the following, we denote the single spin Hilbert space by $\mathcal{H}_{1}$, of dimension $d=2 s+1$. Spins interact with each other according to some Hamiltonian $H=\sum_{\lambda} h_{\lambda}$, where $\lambda$ denotes some sets of spins. As mentioned above, we will introduce different renormalization procedures whose aim is to reduce the number of degrees of freedom by putting together some spins and applying certain operators to neighboring blocks of spins. 


\section{Matrix Product States}

The standard Wilson Renormalization Group ( $R G$ ) procedure as applied to quantum impurity models can be viewed as follows [1, 73]. We take the first two spins, and consider a subspace of the corresponding Hilbert space, $\mathcal{H}_{2} \subset \mathcal{H}_{1} \otimes \mathcal{H}_{1}$ of dimension $d_{2} \leq d_{1}^{2}$. Now we add the next spin, and consider a subspace of the Hilbert space of the three spins, $\mathcal{H}_{3} \subset \mathcal{H}_{2} \otimes \mathcal{H}_{1}$, of dimension $d_{3} \leq d_{2} d_{1}$. We proceed in the same way until we obtain $\mathcal{H}_{N} \subset \mathcal{H}_{N-1} \otimes \mathcal{H}_{1}$, and $d_{N} \leq d_{N-1} d_{1}$. Now, we approximate the Hamiltonian $H$ by $P_{N} H P_{N}$, where $P_{N}$ is the projector onto $H_{N}$. If we can carry out this procedure, and we choose $d_{N}$ sufficiently small, we will be able to diagonalize this Hamiltonian and find the eigenvalues and eigenvectors.

In order to be able to carry out this procedure in practice for a large number of spins, we have to impose that all dimensions $d_{n} \leq D$, where $D$ is fixed (say, of the order of few hundreds). We will call $D$ the bond dimension. Otherwise we will run out of computer resources. Note that, if we always take $d_{n}=d_{n-1} d_{1}$, we will end up with the original Hilbert space of all the spins, $\mathcal{H}_{1}^{\otimes N}$. Although we will have made no approximation, $d_{n}$ will grow exponentially with $N$, and thus the problem will be untractable as soon as we have few tens of spins.

Before giving explicit recipes about how to properly choose the subspaces at each step, and how to determine the final Hamiltonian, let us show what will be the structure of the eigenvectors of the approximate Hamiltonian $H_{N}$. Let us start with $\mathcal{H}_{2}$, and write an arbitrary orthonormal basis, $\left\{|\beta\rangle_{2}\right\}_{\beta=1}^{d_{2}}$, in this subspace as

$$
|\beta\rangle_{2}=\sum_{n_{1}, n_{2}=1}^{d_{1}} B_{\beta}^{n_{1}, n_{2}}\left|n_{1}\right\rangle_{1} \otimes\left|n_{2}\right\rangle_{1} .
$$

Here, $B_{\alpha}^{n_{1}, n_{2}}$ are the coefficients of the basis vectors in terms of the original basis vectors $|n\rangle \in \mathcal{H}_{1}$. We can always express

$$
B_{\beta}^{n_{1}, n_{2}}=\sum_{\alpha=1}^{d_{1}} A[1]_{\alpha}^{n_{1}} A[2]_{\alpha, \beta}^{n_{2}}
$$

where $A[1]_{\alpha}^{n_{1}}=\delta_{n_{1}, \alpha}$ and $A[2]_{\alpha, \beta}^{n_{2}}=B_{\beta}^{\alpha, n_{2}}$. The orthonormality of the vectors (11), together with their definitions, implies that

$$
\sum_{n_{1}=1}^{d_{1}} A[1]_{\alpha}^{n_{1}} \bar{A}[1]_{\alpha^{\prime}}^{n_{1}}=\delta_{\alpha^{\prime}, \alpha}, \quad \sum_{\alpha, n_{2}=1}^{d_{1}} A[2]_{\alpha, \beta}^{n_{2}} \bar{A}[2]_{\alpha, \beta^{\prime}}^{n_{2}}=\delta_{\beta^{\prime}, \beta},
$$

where the bar denotes complex conjugate. We can now write any orthonormal basis in $\mathcal{H}_{3}$ in terms of linear combinations of vectors $|\beta\rangle_{2} \otimes\left|n_{3}\right\rangle$, and proceed in the same way iteratively. After $M$ steps, we have the relation

$$
|\beta\rangle_{M}=\sum_{\alpha=1}^{d_{M-1}} \sum_{n_{M}=1}^{d_{1}} A[M]_{\alpha, \beta}^{n_{M}}|\alpha\rangle_{M-1} \otimes\left|n_{M}\right\rangle_{1}
$$


The orthonormality condition implies

$$
\sum_{\alpha, n_{M}} A[M]_{\alpha, \beta}^{n_{M}} \bar{A}[M]_{\alpha, \beta^{\prime}}^{n_{M}}=\delta_{\beta^{\prime}, \beta},
$$

Substituting recursively the definitions of $|\alpha\rangle_{k}$ in (4), we can write

$$
|\beta\rangle_{N}=\sum_{n_{1}, \ldots, n_{N}=1}^{d_{1}}\left(A_{1}^{n_{1}} A_{2}^{n_{2}} \ldots A_{N}^{n_{N}}\right)_{\beta}\left|n_{1}, n_{2}, \ldots, n_{N}\right\rangle .
$$

Here, we have defined a set of matrices $A_{M}^{n} \in M_{d_{M-1}, d_{m}}$ with components $\left(A_{M}^{n}\right)_{\alpha, \beta}:=$ $A[M]_{\alpha, \beta}^{n}$ which, according to (5), fulfill

$$
\sum_{n=1}^{d_{M}} A_{M}^{n \dagger} A_{M}^{n}=\mathbf{1}
$$

i.e. the $A$ 's are isometries. More specifically, when considering the matrix $V$, of indices

$$
V_{(n, \alpha) \beta}=A_{\alpha, \beta}^{n},
$$

where $(n \alpha)$ is taken as a single index, $V^{\dagger} V=\mathbf{1}$. Note that, since $A_{1}^{n_{1}}$ are (row) vectors and the rest of the $A$ 's are matrices, the product $A_{1}^{n_{1}} A_{2}^{n_{2}} \ldots A_{N}^{n_{N}}$ is a vector, from which we take the $\beta$-th component in (6). The elements of the orthonormal basis in $\mathcal{H}_{N}$, and therefore all the vectors therein, can be expressed in the form (6) : their coefficients in the original basis $\left|n_{1}, \ldots, n_{N}\right\rangle$ can be written as product of matrices. Vectors of that form are termed matrix product states [15] due to their structure. In particular, any state in the coarse-grained subspace is a MPS which we will write as

$$
|\Psi\rangle_{N}=\sum_{n_{1}, \ldots, n_{N}=1}^{d_{1}} A_{1}^{n_{1}} A_{2}^{n_{2}} \ldots A_{N}^{n_{N}}\left|n_{1}, n_{2}, \ldots, n_{N}\right\rangle .
$$

Here, $A_{N}^{n_{N}}$ is a (column) vector fulfilling (7), i.e.

$$
\sum_{n=1}^{d_{1}} \sum_{\alpha=1}^{d_{N-1}}\left|\left(A_{N}^{n}\right)_{\alpha}\right|^{2}=1
$$

Every MPS is invariant under the exchange of $A_{M}^{n} \rightarrow X_{M-1} A_{M}^{n} X_{M}^{-1}$, where the $X$ are non-singular matrices, as it can be checked by direct inspection of (9). This gives us the possibility of choosing a gauge, and thus impose conditions to the matrices $A$ which simplify the further calculations, or which give a physical meaning. In our case, we can consider (77) as a gauge condition which implements such a choice and makes a direct connection between MPS and the renormalization group method.

\subsection{Expectation values}

The whole renormalization procedure is completely determined by the matrices $A_{M}^{n} \in$ $M_{d_{M-1}, d_{M}}$. Before giving different prescriptions on how to determine them, we will show 
how to calculate expectation values of different observables [14]. Let us consider first $X=\sigma_{1} \otimes \sigma_{2} \otimes \ldots \sigma_{N}$, where the sigma's are operators acting on $\mathcal{H}_{1}$. Using (9), we have

$$
\langle\Psi|X| \Psi\rangle=\sum_{n_{k}, m_{k}=1}^{d_{1}} \prod_{k}\left\langle m_{k}\left|\sigma_{k}\right| n_{k}\right\rangle C_{n_{1}, \ldots, n_{M}, m_{1}, \ldots, m_{N}}
$$

Here,

$$
C_{n_{1}, \ldots, n_{M}, m_{1}, \ldots, m_{N}}=A_{1}^{n_{1}} \ldots A^{n_{N}} \bar{A}_{1}^{m_{1}} \ldots \bar{A}_{N}^{m_{N}}=\left(A_{1}^{n_{1}} \otimes \bar{A}_{1}^{m_{1}}\right) \ldots\left(A_{N}^{n_{N}} \otimes \bar{A}_{N}^{m_{N}}\right)
$$

Defining

$$
E_{M}\left[\sigma_{M}\right]:=\sum_{n, m=1}^{d_{1}}\left\langle m\left|\sigma_{M}\right| n\right\rangle A_{M}^{n} \otimes \bar{A}_{M}^{m}
$$

we have

$$
\langle\Psi|X| \Psi\rangle=E_{1}\left[\sigma_{1}\right] E_{2}\left[\sigma_{2}\right] \ldots E_{N}\left[\sigma_{N}\right] .
$$

Thus, the expectation value has a very simple expression: it is itself a product of matrices $\mathcal{S}$. Typically, one is interested in few-body correlation functions, in which case most of the $\sigma$ 's are the identity operator. For those cases we will denote $E[\mathbf{1}]=: E$. In particular, the normalization of $|\Psi\rangle$ can be written as $\langle\Psi \mid \Psi\rangle=E_{1} E_{2} \ldots E_{N}=1$. This can be readily checked by using (3, 13) and writing the vector $E_{1}$ as $\left(\Phi_{1} \mid\right.$, where

$$
\left.\left.\mid \Phi_{M}\right):=\sum_{\alpha=1}^{d_{M}} \mid \alpha, \alpha\right),
$$

and the $\mid \alpha$ ) are orthonormal vectors $Џ$. Besides that, the orthonormality condition (5) immediately implies $\left(\Phi_{M-1} \mid E_{M}=\left(\Phi_{M} \mid\right.\right.$ and thus $E_{1} E_{2} \ldots E_{N}=1$ [cf. (10)] $)$.

\subsection{Real-space renormalization group}

One of the simplest prescriptions to carry out the renormalization procedure is to choose the orthonormal basis $|\beta\rangle_{M}$ as we proceed [1, 5. Since we are interested in the low energy sector of our Hilbert space at each step, $\mathcal{H}_{M}$, the natural choice is to take the $d_{M}$ lowest energy states.

This construction becomes simpler if we have a Hamiltonian with nearest-neighbor interactions only, i.e.

$$
H=\sum_{M=2}^{N} h_{M}
$$

where $h_{n}$ acts on spins $M-1$ and $M$, i.e.

$$
h_{M}=\sum_{k} \tilde{h}_{M}^{k, l} \sigma_{M-1}^{k} \otimes \sigma_{M}^{l} .
$$

$\mathcal{S}$ Note that $E_{1}$ and $E_{N}$ are a row and a column vector, respectively, and the rest matrices

$\|$ Note that they are in the space where the matrices $A$ act, and not in the Hilbert spaces $\mathcal{H}$ corresponding to the spins 
We start out by choosing as $|\beta\rangle_{2}$ the $d_{2}$ eigenstates of $h_{2}$ with the lowest energy, $e_{\beta}^{2}$. We can write

$$
h_{2} \simeq \sum_{\beta=1}^{d_{2}} e_{\beta}^{2}|\beta\rangle\langle\beta| .
$$

In the $M$-th step, once we have determined the basis $|\beta\rangle_{M-1}$ in the previous one $(M>2)$, we write

$$
H_{M}=\sum_{n=2}^{M} h_{n}
$$

in the basis $|\beta\rangle_{M-1} \otimes\left|n_{M}\right\rangle$. This is very simple, since we already have up to $h_{M-1}$ from the previous step, and we thus just have to do that for $h_{M}$. Inspecting (17) it is clear that, apart from the matrix elements of $\sigma_{M}^{l}$ in the basis $|n\rangle$, we just have to determine the matrix elements of any operator acting on the $(M-1)$ spin in the basis $|\beta\rangle_{M-1}$. But this can be done right away, since

$$
\left\langle\beta^{\prime}|\sigma| \beta\right\rangle=\sum_{n=1}^{d_{1}} \sum_{\alpha=1}^{d_{M-2}}\langle m|\sigma| n\rangle\left(A_{M-1}^{n}\right)_{\alpha, \beta}\left(\bar{A}_{M-1}^{m}\right)_{\alpha, \beta^{\prime}} .
$$

Once we finish this procedure we will end up with an orthonormal basis in $\mathcal{H}_{N}$, which should reproduce the low energy sector of the Hamiltonian $H$.

\subsection{Density matrix renormalization group}

Starting from the previous discussion, we know that any real-space renormalization procedure will give rise to a MPS. Thus, the best approximation to the ground state of our Hamiltonian will be obtained using a variational principle. That is, by minimizing the energy $e=\langle\Psi|H| \Psi\rangle$ with respect to all MPS of the form (91). For that, we can just use the expression (14) and write

$$
\langle\Psi|H| \Psi\rangle=\sum_{M=2}^{N} \sum_{k, l} \tilde{h}_{M}^{k, l} E_{1} E_{2} \ldots E_{M-1}\left[\sigma^{k}\right] E_{M}\left[\sigma^{l}\right] E_{M+1} \ldots E_{N} .
$$

This formula explicitly shows the dependence of the energy on the matrices $A_{M}^{n}$, and thus, in principle, can be used to determine those matrices which minimize it. One possible strategy is to minimize sequentially with respect to all possible $A$ 's. That is, we fix all $A_{M}^{n}$ for $M>2$, so that (21) is a function of $A_{2}^{n}$ only 9 . This functional dependence appears only in $E_{2}$ and $E_{2}\left[\sigma^{k}\right]$. Actually, if we write (21) explicitly in terms of $\left(A_{2}^{n}\right)_{\alpha, \beta}$, we see that such a dependence is quadratic. We also have to impose the normalization condition (7), which in turn implies the normalization of $|\Psi\rangle$, and which gives quadratic equations on those coefficients as well. Thus, minimizing the energy at this point consists of minimizing a quadratic polynomial with quadratic constraints. Once we have $A_{2}^{n}$, we minimize with respect to $A_{3}^{n}$ by fixing the rest of the $A^{\prime}$ 's. We proceed in the same vein until we reach $A_{N}^{n}$. At this point, we continue with $A_{N-1}^{n}$ and

I Note that $A_{1}^{n}$ is always fixed. 
so on. That is, we sweep the spins from left to right, to left, etc, determining at each step the matrices associated to that particular spin. At each step, the energy is smaller or equal than the one considered in the previous step. This can be easily understood since at that step we do not vary the matrices $A$ at the site we are minimizing, we will obtain the previous energy. Thus, minimizing with respect to the $A$ 's cannot increase the energy. Consequently, this procedure must converge to a minimum of the energy $\square$ More specifically, each step consists of minimizing $\langle\Psi|H| \Psi\rangle /\langle\Psi \mid \Psi\rangle$. Both numerator and denominator are quadratic functions of the $A_{m}^{n}$ if we fix the rest. That is, if we write a long vector $x_{M}$ containing all the coefficients of $A_{M}^{1}, A_{M}^{2}, \ldots, A_{M}^{d_{1}}$, we can write

$$
e=\frac{x_{M}^{\dagger} H_{M} x_{M}}{x_{M}^{\dagger} N_{M} x_{M}},
$$

where $H_{M}$ and $N_{M}$ can be determined using (21). It follows from the hermiticity of $H$ that $H_{M}$ is hermitian. Furthermore, $N_{M}$ is positive semidefinite (as the norm of a vector must be non-negative). Thus, $e$ is real and its minimum can be determined through the generalized eigenvalue equation

$$
H_{M} x_{M}=\lambda N_{M} x_{M}
$$

where one has to choose the minimum $\lambda$ fulfilling that. From this equation, we also determine $x_{M}$ and thus $A_{M}^{n}$. The only drawback is that the matrix $N_{M}$ may be illconditioned, something which may pose some problems when solving Eq. (23).

There is a way to circumvent this misfortune and, at the same time, simplify the algorithm further. As mentioned above, we have that $\left(\Phi_{M-1} \mid E_{M}=\left(\Phi_{M} \mid\right.\right.$, a fact that can be used to simplify Eq. (21). Actually, we could have started our renormalization procedure from the $N$-th spin in decreasing order. In that case, instead of (5,77) we would have had $\left.E_{N}=\mid \Phi_{1}\right)$,

$$
\sum_{n=1}^{d_{M}} A_{M}^{n} A_{M}^{n \dagger}=\mathbf{1}
$$

and thus $\left.\left.E_{M} \mid \Phi_{M+1}\right)=\mid \Phi_{M}\right)$. This suggests a mixed strategy, where we renormalize in increasing order up to the spin $M-1$, and in decreasing one up to $M+1$, whenever we are minimizing the energy in site $M$. In this case we have

$$
\begin{aligned}
\langle\Psi \mid \Psi\rangle & =E_{1} \ldots E_{M-1} E_{M} E_{M+1} \ldots E_{N}=\left(\Phi\left|E_{M}\right| \Phi\right) \\
& =\sum_{\alpha, \beta=1}^{d_{M-1}, d_{M}} \sum_{n=1}^{d_{1}}\left|\left(A_{M}^{n}\right)_{\alpha, \beta}\right|^{2} .
\end{aligned}
$$

That is, the matrix $N_{M}$ is simply the identity matrix, and therefore Eq. (23) becomes a standard eigenvalue equation. Once we have determined $A_{M}^{n}$ by solving it, and if we are going to minimize next the matrices at site $M+1$, we write $A_{M}^{n}=U_{M}^{n} X$, where $U_{M}^{n}$ is an isometry [i.e. fulfills (7), cf. (8) ]. For instance, we define $V$ according to (8), and use the singular value decomposition to write $V=U D W$, where $U$ and

+ This can be a local or a global minimum. 
(a)

(b)

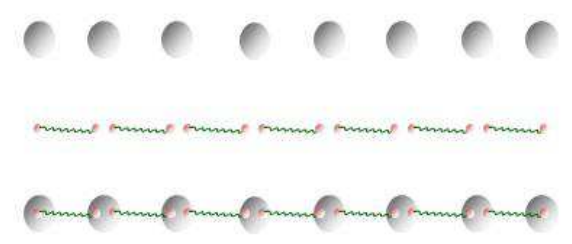

Figure 1. Construction of a MPS in terms of entangled auxiliary particles. (a) Original spin system; (b) We replace each spin by two auxiliary particles (except at the ends of the chain), which are in a maximally entangled state with their neighbors; (c) The final state is obtained after mapping the state of each pair of auxiliary particles locally onto the original spins.

$W$ are isometries and $D \geq 0$ is diagonal, i.e. $X=D W$. We can now discard the $X$ and keep $U_{M}^{n}$ for site $M$, since we can always take $A_{M+1}^{n} \rightarrow X A_{M+1}^{n}$, so that the state $|\Psi\rangle$ remains the same. Given that we are going to optimize now anyway with respect to $A_{M+1}^{n}$, we can ignore the multiplication by $X$. In this way, we make sure that $\left(\Phi_{M-1} \mid E_{M}=\left(\Phi_{M} \mid\right.\right.$, which is consistent with our procedure. In case we are going to minimize next the matrices at site $M-1$, we just have to decompose $A_{M}^{n}=X U_{M}^{n}$, where now $U_{M}^{n}$ fulfills (24). Apart from that, it is numerically convenient to store the values of $E_{k}[\sigma] E_{k+1}\left[\sigma^{\prime}\right] E_{k+2} \ldots E_{M-1}$ and $E_{M-1}[\sigma]$, for the $\sigma$ 's that appear in the Hamiltonian, and update them when we have determined $A_{M}^{n}$. Besides, one should also store those of the form $E_{M+1} \ldots E_{k-1} E_{k}[\sigma] E_{k+1}\left[\sigma^{\prime}\right]$ as well as $E_{M+1}[\sigma]$, since they will be needed in future optimizations.

The algorithm carried out in this way is (up to minor modifications) the celebrated density matrix renormalization group algorithm introduced by S. White in 1991 [3, 4]. The only minor differences are: (i) he optimized two sites at the same time, say $M$ and $M+1$ and from there he determined $A_{M}^{n}$ by using a singular value decomposition; (ii) he proposed a method to determine the initial configuration of the $A$ 's by growing the number of spins until the desired value $N$ is reached. Furthermore, he derived his algorithm from a very intuitive method in which he described the effect of the rest of the spins on any given one in the normalization in an efficient way. In any case, the present discussion highlights the variational character of (the finite version of) DMRG [10].

\subsection{Matrix Product States and Projected Entangled-Pair States}

The MPS can also be introduced from a different perspective, which highlights their entanglement content and is amenable to several generalizations. The idea is to extend the AKLT construction [12, where one substitutes the original spins by couples of auxiliary systems in a prescribed state, and then projects their state back to the spin Hilbert space [44, 43].

For the definition and construction of the state, we imagine that each site of the spin chain, $M$, we have two ancillas $l_{M}, r_{M}$, with corresponding Hilbert spaces of dimensions $d_{M-1}$ and $d_{M}$, respectively (see Fig. 1(a)). For $M=1(M=N)$ we have only one 
ancilla $r_{1}\left(l_{N}\right)$. The state of the ancillas is fixed: they are maximally entangled to their nearest neighbor (see Fig. 1(b)). That is, $r_{M-1}$ and $l_{M}$ are in a state $\left.\mid \Phi_{M-1}\right)^{*}$ Now, in order to recover the MPS, we map the state of the ancillas onto the one of the spins at each site (Fig. 1(c)). That is, we write

$$
\left.\left.|\Psi\rangle=P_{1} \otimes \ldots P_{N} \mid \Phi_{1}\right) \otimes \ldots \mid \Phi_{N-1}\right)
$$

where $P_{M}: \mathcal{H}_{M-1} \otimes \mathcal{H}_{M} \rightarrow \mathcal{H}_{1}$. We write each of those maps in the bases we have chosen for each Hilbert spaces

$$
P_{M}=\sum_{n=1}^{d_{1}} \sum_{\alpha=1}^{d_{M-1}} \sum_{\beta=1}^{d_{M}}\left(A_{M}^{n}\right)_{\alpha, \beta}|n\rangle(\alpha, \beta \mid .
$$

It is a simple exercise to show that, indeed, the state defined in (26) coincides with that of (9). Thus, MPS can be obtained by projecting entangled-pairs of ancillary particles onto the spaces of the spins, thus the name 1-dimensinal PEPS.

This construction allows us to derive a variety of the properties of MPS right away. First of all, if we consider the reduced density operator, $\rho_{1,2, \ldots, M}$, of the first $M$ spins, its rank (number of non-zero eigenvalues) is bounded by $d_{M}$. The reason is that the original state of the ancillas obviously fulfills that condition, and that the rank cannot increase by applying any operator, in particular $P_{1} \ldots \ldots P_{M}$. This implies that the von-Neumann entropy $S\left(\rho_{1,2, \ldots, M}\right) \leq \log _{2} d_{M} \mathbb{H}$, i.e. the entropy of a block of contiguous spins is bounded by the maximum value of $d_{M}$. The fact that the entropy of a block of spins is bounded (for infinite chains) is sometimes referred as the area law, as mentioned in the introduction.

Apart from that, we can also prove that any spin state can be written as a MPS. For that, consider that $l_{M}\left(r_{M}\right)$ is, in turn, composed of $N-M+1(N-M)$ spins, as sketched in Fig. 1. Now, for $M>1$ the map

$$
P_{M}=\left(\left.\Phi_{2}\right|^{\otimes N-M} \otimes \sum_{n=1}^{d_{1}}|n\rangle(n \mid\right.
$$

teleports [74] the state of the first $N-M$ spins of $l_{M}$ to $r_{M+1}$, while leaving the last one as the physical spin. By choosing

$$
P_{1}=\sum|n\rangle\left(\Psi_{n} \mid \otimes(n \mid\right.
$$

where $\left|\Psi_{n}\right\rangle={ }_{1}\langle n \mid \Psi\rangle$, we have the desired result.

\subsection{Translationally invariant systems}

We mention now the possibility of dealing with systems with periodic boundary conditions corresponding, for instance, to translationally invariant Hamiltonians. A

* We have used $\mid . .$.$) to denote states on the Hilbert space of the ancillas. As it will be clear, this is a$ natural choice in view of our definition (15).

$\sharp$ We take $S(\rho)=-\operatorname{tr}\left[\rho \log _{2} \rho\right]$ 
MPS which automatically fulfills this condition is the one in which the matrices $A$ at different sites are the same. In that case, we can write

$$
|\Psi\rangle_{N}=\sum_{n_{1}, \ldots, n_{N}=1}^{d_{1}} \operatorname{tr}\left[A^{n_{1}} A^{n_{2}} \ldots A^{n_{N}}\right]\left|n_{1}, n_{2}, \ldots, n_{N}\right\rangle .
$$

This class of states, in the limit $N \rightarrow \infty$, appeared even before the name MPS was coined, and were called finitely correlated states[14]. They were introduced when extending the 1D version of the AKLT model [12], whose ground state is the most prominent example of a state in that clase. They were also viewed as a systematic way of building translationally invariant states [14. States with different matrices were not considered at that time since the emphasis was given to infinite translationally invariant spin chains. Note also, that any translationally invariant state may be written in this form [17].

The matrices $E_{M}=: E$ now coincide and $E$ is called transfer matrix, given the analogy of the formulas with those of classical statistical mechanics. Its eigenvalues reflect the correlation length and other properties of the system. This can be understood since if we consider the two-spin (connected) correlation functions at distance $L$, the matrix $E^{L}$ will appear in the calculation. In the limit $L \gg 1$ only the largest eigenvalues of $E$ will give a contribution to the correlation function, which will thus decay exponentially. Thus, the name finitely correlated states used for states of the form (30) in the limit $N \rightarrow \infty$ [14].

Let us show that, without loss of generality, we can impose the Gauge condition (7). For that, we denote by $X$ the operator corresponding to the largest eigenvalue $\lambda$ (in absolute value) of the following eigenvalue equation

$$
\sum_{n} A^{\dagger n} X A^{n}=\lambda X
$$

One can can always choose $\lambda=1$ by re-scaling and $X=X^{\dagger}>0$. Then, the matrices $\tilde{A}^{n}:=X^{1 / 2} A^{n} X^{-1 / 2}$ are well defined, correspond to the same state as that of $A^{n}$, and fulfill the Gauge condition.

We consider now the renormalization procedure in this particular case. Instead of performing that step by step, as explained above, we can aim at minimizing the energy directly within the MPS of the form (30). For that, we just have to specialize (30), and consider a single term in the Hamiltonian (17) given the translational symmetry, i.e.

$$
\frac{\langle\Psi|H| \Psi\rangle}{\langle\Psi \mid \Psi\rangle}=N \sum_{k, l} \tilde{h}_{M}^{k, l} \frac{\operatorname{tr}\left(E\left[\sigma^{k}\right] E\left[\sigma^{l}\right] E^{N-2}\right)}{\operatorname{tr}\left(E^{N}\right)}
$$

Note that, as opposed to the previous case (where we had open boundary conditions), the Gauge condition does not guarantee that the state $|\Psi\rangle$ is normalized. The energy so defined is a function of the $A$ 's, so that we can aim at minimizing this expression directly. The latter simplifies in the limit $N \rightarrow \infty$ if the maximum eigenvalue of $E$ is not degenerate,

$$
\lim _{N \rightarrow \infty} \frac{\langle\Psi|H| \Psi\rangle}{N\langle\Psi \mid \Psi\rangle}=\frac{1}{\lambda} \sum_{k, l} \tilde{h}_{M}^{k, l}\left\langle L\left|E\left[\sigma^{k}\right] E\left[\sigma^{l}\right]\right| R\right\rangle .
$$


Here $|L\rangle$ and $|R\rangle$ are the right and left eigenvectors corresponding to the maximum eigenvalue $\lambda$

$$
E|R\rangle=\lambda|R\rangle, \quad\langle L| E=\lambda\langle L| .
$$

Here we have not imposed the Gauge conditions, in which case we would have $\lambda=1$. The minimization of (33) with respect to $A^{n}$ can be now performed directly using, eg, using conjugate gradient methods.

The variational method exposed above was first proposed by Rommer and Ostlund in the context of DMRG [7, 75] (see also [8]). They realized that, in the infinite version [3], if $N \rightarrow \infty$, the fixed point of the DMRG procedure will correspond to a MPS with all matrices equal (ie a finitely correlated state). Then they suggested to take those states as variational states and minimize the energy using standard methods. Alternatively, one may perform the minimization by using evolution in imaginary time applied to an infinite system [76]. It is not strictly necessary to take all the matrices $A$ identically (i.e. ...AAAA ...) [71], but it may be more convenient to alternate two kinds of matrices (i.e. ...ABAB ...)[76]. Note that this case would be included if we group pairs of neighboring spins and take identical matrices in each group $(C C C C \ldots)$, since this covers the previous case if we take $C=A B$. In practice, however, the latter approach may be less efficient numerically since one has to deal with larger spins.

\subsection{Graphical representation}

When using many-particle quantum states, we reach very soon a cumbersome level of notation. This is not an exception when we utilize the language of MPS, since we typically have products of many matrices, which depend on another index that corresponds to each individual spin. As soon as we express expectation values of observables, the notation gets very involved. There is a simple way of conveying the same information by using a graphical representation of MPS, which we will introduce here and that will be used in the following sections. For that, let us consider the $A$ 's describing the MPS as a rank three tensor $\left(A_{\alpha, \beta}^{n}\right.$ of indices $\left.n, \alpha, \beta\right)$, as shown in Fig. 2(a). These tensors are contracted along the indices $\alpha, \beta$ to form the matrix product state (Fig. 2(b)). More precisely, the $\left\langle n_{1}, \ldots, n_{N} \mid \Psi\right\rangle$ is obtained after this contraction, where the indices $n$ are still open. We can thus represent the MPS as the tensor of Fig. 2(c). If the first and the last objects are also rank three tensors, we will have the representation of Fig. 2(d), which in turn describes, eg, a translationally invariant state. Any local observable, $\sigma$, can be represented as a tensor itself, if we write it in the spin basis (Fig. 2(e)).

The norm of the state can be obtained by tracing the tensor with respect to

the spin indices. This is represented in Fig. 2(f.1), where the upper part represents $\left\langle n_{1}, \ldots, n_{N} \mid \Psi\right\rangle$ and the lower the complex conjugate, and the indices $n$ are contracted. By considering each pair of tensors $A$ and $\bar{A}$ on top of each other, we can build the matrix $E[\mathbf{1}]$ defined in (13), and thus represent the norm as the contraction of those 
(a)

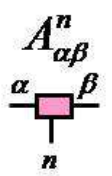

(e)

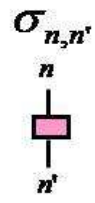

(f.1)

(f. 2)

$\langle\Psi \mid \Psi\rangle$ (b)

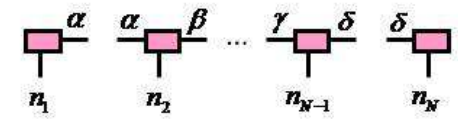

(c)

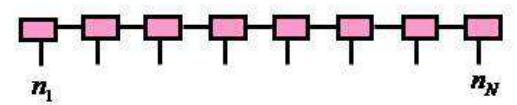

(d)

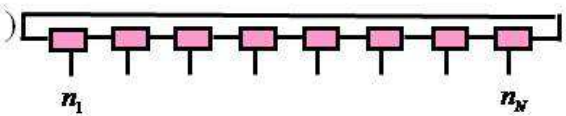

$n_{1}$
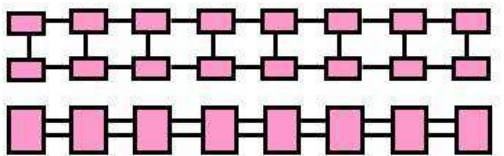

(g. 1$)\left\langle\Psi\left|\sigma_{2} \sigma_{4}\right| \Psi\right\rangle$

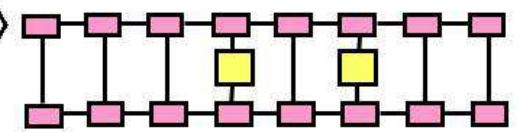

(g.2)

Figure 2. Graphical representation of an MPS in terms of contracted tensors (tensor network). (a) The set of matrices $A^{n}$ are represented in terms of a rank-3 tensor where the index $n$ is pointing vertically; (b) We consider the set of tensors corresponding to each spins and (c) contract them according to the horizontal indices; (d) the same can be done with periodic boundary conditions by adding an extra bond on the end spins; (e) Tensor representation of an operator acting on a spin; (f.1) In order to calculate $\langle\Psi \mid \Psi\rangle$ we contract the tensor corresponding to $\Psi$ with that of $\bar{\Psi}$, giving rise to (f.2) a row of tensors which are contracted to give a number. The tensors can be viewed as matrices (one double-index to the left and another to the right). (g.1) and (g.2) the same but with an expectation value.

matrices (Fig. 2(f.2), compare (14)). In the same way, we can represent expectation values of product of local observables (Figs. 2(g.1) and (g.2)).

\subsection{Sequential generation of Matrix Product States}

We have seen so far that the family of MPS corresponds to those that appear in real space renormalization schemes. Here we will show that they also coincide with the states that can be sequentially generated[59]. For that, let us assume first that we have an auxiliary system, i.e an ancilla (which, in practice, could be a $D$-level atom) with Hilbert space $H_{a}$ of dimension $D$, initially prepared in state $|1\rangle$, and also all the spins in the chain in state $|1\rangle$. Now we consider a unitary operation between the ancilla and the first spin, then between the ancilla and the second on, and so on, until the ancilla interacts with last spin (see Fig. 3(a)). Let us denote by $U^{(A, M)}$ the unitary operation 
(a)

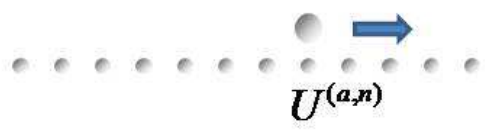

(b)

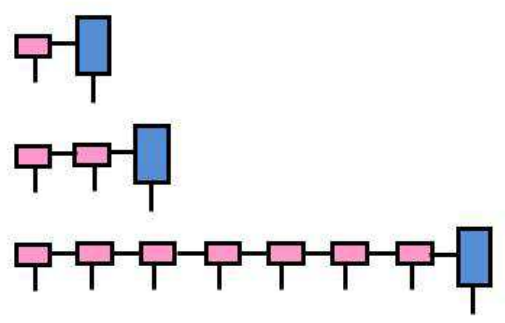

(c)

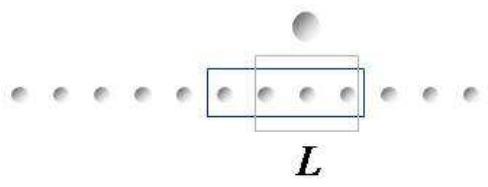

Figure 3. Sequential generation of MPS. (a) Using an ancilla with Hilbert space of $D$ dimensions, we act sequentially on the first, second, etc spins with unitary operators; (b) This process can be understood with the graphical language introduced before. After each interaction, the spins get entangled in a MPS with the ancilla itself. (c) We can replace the ancilla by the $\log D$ spin which are to the right of the spin we are acting on.

between the ancilla and the $n$-th spin. If we now denote by $\mid \alpha$ ) an orthonormal basis in the ancilla Hilbert space, and write

$$
\left.U^{(A, M)}|0\rangle_{1}=\sum_{\alpha, \beta=1}^{D} \sum_{n=1} A[M]_{\alpha, \beta}^{n}|n\rangle_{1} \mid \alpha\right)(\beta \mid,
$$

then we end up with the state

$$
\left.|\Psi\rangle_{N}=\sum_{n_{1}, \ldots, n_{N}=1}^{d_{1}}\left(A_{1}^{n_{1}} A_{2}^{n_{2}} \ldots A_{N}^{n_{N}}\right)_{\beta}\left|n_{1}, n_{2}, \ldots, n_{N}\right\rangle \mid \beta\right) .
$$

In Fig. 3(b) we have used the representation introduced above to describe this process and to arrive at the above formula.

From Eq.(36) it also immediately follows that any MPS can be created sequentially using an ancilla, by simply choosing the $U^{(A, M)}$ according to (35), and the last one in such a way that $A_{N}^{n_{N}}$ is a vector (ie it only has one component $\beta=1$ ). Besides, we can substitute the ancillary particle at each step by $L \geq \log _{d_{1}} D$ spins which lie on the right of the particle we are acting on, since they span a Hilbert space which has dimension at 
least $D$. Once we apply $U^{A 1}$ to the first $L+1$ spins, we can swap the state of the spins $2, \ldots, L+1$ to $3, \ldots, L+2$ by using a unitary operation acting on spins $2, \ldots, L+2$. Now we can use the spins $3, \ldots, L+2$ as ancilla, and apply $U^{A 2}$ to the second spin plus those. Proceeding in this way, we see that we can also prepare any MPS by a sequence of unitary operations from left to right, each of them acting on at most $L+1$ spins (see Fig. [3(c)).

\subsection{Approximating states with Matrix Product States}

Given a state, $|\Phi\rangle$, expressed in a given basis, how do we find its MPS representation? This is very simple in theory, since we just have to follow the procedure of the renormalization, but keeping all the states that are necessary. We can do that as follows. We first consider the reduced density operator of the first spin, $\rho_{1}=\operatorname{tr}(|\Phi\rangle\langle\Phi|)$ and a basis, say $|\beta\rangle_{1}$, where it is diagonal. Then we consider the first two spins, and do the same with the reduced density operator $\rho_{2}$, determining a basis $|\beta\rangle_{2}$. Obviously, we can write (11). We continue in the same way, so that at the end we can write the state $|\Phi\rangle$ as in (6), and thus as a MPS.

This procedure, in general, will not work with a large number of particles since we will have to diagonalize matrices $\left(\rho_{M}\right)$ of dimensions that grow exponentially with the number of spins, and thus the matrices $A$ will also be too large to be handled (Note that if the rank of all operators $\rho_{M}$ is smaller than some fixed number, say $D$, the procedure will express the state as a MPS with maximal bond dimension $D$. In other words, any state can be written as a MPS of bond dimension equal to the maximal rank of the reduced density operators $\rho_{M}$ ). One way to circumvent this problem is to look for a good approximation to the state $|\Phi\rangle$ in terms of a MPS, $|\Psi\rangle$. One can do that using the same idea as the renormalization procedures presented above:

- Real-space-like approximation: [77] Here, every time we diagonalize $\rho_{M}$, we only pick $D$ states $|\alpha\rangle_{M}$, those eigenvectors corresponding to the $D$ largest eigenvalues of $\rho_{M}$. In this way, as in the real-space renormalization procedure, we try at each step to be as close as possible to the state $|\Phi\rangle$.

- DMRG-like approximation: 47] Instead of trying to optimize locally, at each step, the subspaces we select in order to represent $\Phi$, we can do something better. The inspiration comes from DMRG, where one does not perform an optimization locally, but more globally. We can do the same thing here, ie to obtain the $A$ 's that approximate the state $|\Phi\rangle$ variationally, so that they provide the best possible approximation. In practice, this means that we maximize $|\langle\Psi|P| \Psi\rangle|$, where $P=|\Phi\rangle\langle\Phi|$. This minimization is, in turn, similar to the minimization of the energy of the ground state of $H$, cf. Eq. (21). Thus, we can follow the same procedure, namely sequentially minimize with respect to each of the $A$ 's fixing the rest.

The procedures exposed above will be still hard to implement in practice, due to the

fact hat we still have to deal with too many parameters (those describing $|\Phi\rangle$ ). However, 
if that state is initially written in a MPS form (of matrices with a large but fixed bond dimension, $D$ ) or in a superposition thereof with few terms, then we will be able to do that in practice, since all the operations can be made efficiently. For example, the term $|\langle\Psi|P| \Psi\rangle|$ will be a polynomial of second degree in the coefficients of each particular $A[M]$ that can be easily determined.

The above procedure can be used to simplify and compress a MPS description. For instance, imagine we have a MPS with matrices $A$ of dimension $D$. The goal is to find another MPS which is very close to that one, of matrices $B$ with a smaller dimension, $D^{\prime}$. In practice, this technique can be used for many purposes. For example, to approximate the evolution of a MPS under the action of a Hamiltonian of the form (16), or some quantum gates. The idea in this case is to apply the evolution operator for a short time, such that we can determine the evolution after this step (using, eg, perturbation theory or neglecting terms that do not commute in the Hamiltonian), and then approximate that state with a MPS of a fixed dimension. The real-space approximation explained above gives rise to the celebrated time evolving block decimation (TEBD) algorithm [63, 64, 65, 66] whereas the optimal DMRG-like one was presented in [47].

\subsection{Matrix Product Operators}

In the same way one defines MPS, one can define operators which can be written in terms of products of matrices, the so-called matrix product operators (MPO) [47]. They are of the form

$$
X=\sum_{n_{1}, \ldots, n_{N}=1}^{d_{1}} \operatorname{tr}\left(B_{1}^{n_{1}} B_{2}^{n_{2}} \ldots B_{N}^{n_{N}}\right) O_{n_{1}} \otimes O_{n_{2}} \ldots \otimes O_{n_{N}} .
$$

Here, $\left\{O_{n}\right\}_{n=1}^{d_{1}^{2}}$ forms a basis in the space of operators acting on $\mathcal{H}_{1}$. For instance, we may take $O_{n n^{\prime}}=|n\rangle\left\langle n^{\prime}\right|$.

A class of operators that can be easily written as MPO are Hamiltonians with short-range interactions. The idea is to realize that the space of operators acting on each spin is itself a Hilbert space (now of dimension $d_{1}^{2}$ ), so that all the properties of MPS directly apply to MPO, and this allows us to write the Hamiltonian as a simple MPO. In order to do that explicitly, let us write $|k\rangle=\sigma^{k}$ with $|1\rangle=\mathbf{1}$, so that $h_{M}$ defined in (17) becomes

$$
h_{M}=|1\rangle \otimes \ldots|1\rangle \otimes\left[\sum_{k} \tilde{h}_{M}^{k, l}|k\rangle_{M-1} \otimes|l\rangle_{M}\right] \ldots \otimes|1\rangle .
$$

From this expression it becomes apparent that $h_{M}$ can be written as a MPS with bond dimension, $D \leq d_{1}^{2}$ : when considering $H$, we can take it as a sum of three terms, those corresponding to $M<M_{0}, M>M_{0}$, and $M=M_{0}$. When writing the analog of the reduced density operator, $\rho_{M_{0}}$ for $H$ we see that both the first term and the second one will just give a contribution of one to its rank, whereas the third one gives at most $d_{1}^{2}-2\left(d_{1}-1\right)$ (Note that we can include the terms with $k=1$ or $l=1$ in $h_{M_{0} \mp 1}$, respectively). Thus, $H$ can be written as a MPO with bond dimension $D \leq d_{1}^{2}-2 d_{1}+3$. 
If we write the Hamiltonian (or any other operator) in this form, one can determine expectation values with MPS in a very efficient way (compare (14)):

$$
\langle\Psi|X| \Psi\rangle=\tilde{E}_{1} \tilde{E}_{2} \ldots \tilde{E}_{N},
$$

where

$$
\tilde{E}_{M}=\sum_{n, m, k} A_{M}^{n} \otimes B_{M}^{k} \otimes \bar{A}_{M}^{m}\left\langle m\left|O_{k}\right| n\right\rangle .
$$

This provides an alternative way of determining expectation values of the Hamiltonian, and thus to carry out DMRG calculations.

Another class of operators for which it is useful the MPO description is the one of density operators, $\rho$, describing the full spin chain. Those are self-adjoint and positive semidefinite, something which is not easy to express in terms of the matrices $B$. However, for those we can use the idea of purification: namely, we can always extend our spin chain with another auxiliary one, with the same number of spins, in such a way that the state of both chains, $|\Psi\rangle$, is pure but when we trace the auxiliary one we obtain the original density operator, $\rho$ [47]. We write $|\Psi\rangle$ as an MPS

$$
|\Psi\rangle=\sum_{n_{1}, \ldots, n_{N}=1}^{d_{1}} A_{1}^{n_{1} m_{1}} A_{2}^{n_{2} m_{2}} \ldots A_{N}^{n_{N} m_{N}}\left|n_{1}, n_{2}, \ldots, n_{N}\right\rangle\left|m_{1}, m_{2}, \ldots, m_{N}\right\rangle .
$$

Thus, we obtain $\rho$ in the form of a MPO (37) with $O_{n n^{\prime}}=|n\rangle\left\langle n^{\prime}\right|$ and

$$
B_{M}^{n n^{\prime}}=\sum_{m=1}^{d_{1}} A_{M}^{n, m} \otimes \bar{A}_{M}^{n^{\prime} m}
$$

Now, we can use the methods described in previous sections to determine the time evolution of a density operator (either directly using the MPO description, or using the purified state). In particular, we can describe the thermal equilibrium state as

$$
e^{-\beta H}=e^{-\beta H / 2} \mathbf{1} e^{-\beta H / 2},
$$

where 1 can be trivially expressed in terms of a purification. Thus, by performing the time evolution (in imaginary time) starting from the purification of $\mathbf{1}$ up to a time $t=i \beta / 2$, we obtain the desired MPO.

Other examples of MPO are transfer matrices in classical systems, as well as the monodromy matrices as appearing in the algebraic Bethe Ansatz [78].

\section{Tree Tensor States}

Another way of carrying out the renormalization procedure in one dimension is to follow Kadanoff's original idea [2]. Let us assume, for simplicity, that $N$ is a power of 2. We split our $N$ spins into $N / 2$ neighboring pairs. For each pair, we consider a subspace $\mathcal{H}_{2}^{\mathrm{k}} \subset \mathcal{H}_{1} \otimes \mathcal{H}_{1}$ of dimension $d_{2}^{\mathrm{k}}$. The resulting systems are paired again into $N / 4$ neighboring couples, where we take $\mathcal{H}_{3}^{\mathrm{k}} \subset \mathcal{H}_{2}^{\mathrm{k}} \otimes \mathcal{H}_{2}^{\mathrm{k}}$ of dimension $d_{3}^{\mathrm{k}}$, and continue in the same vein until we end up with a single system. The comparison of this way of 
(a)

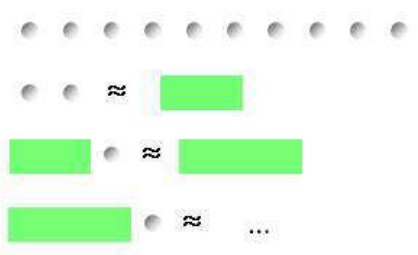

(b)

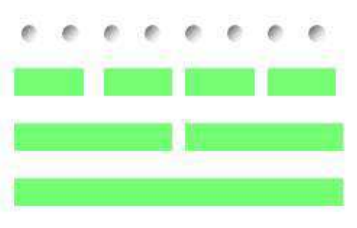

Figure 4. Comparison of the two renormalization procedures. (a) At each step, we add a new spin (ball) to the previous system (square) obtaining a new Hilbert space, which we truncate to obtain the one of a smaller dimension (new square). (b) At each step, we take two neighboring systems (squares) and truncate the Hilbert space to obtain the new one of the new systems.

performing the renormalization and the one given in the previous section is represented in Fig. 4.

As before, we can follow which kind of states are supported in the final subspace $\mathcal{H}_{n}^{\mathrm{k}}$. At the second step, an orthonormal basis in $\mathcal{H}_{2}^{\mathrm{k}}$ corresponding to particles $2 M-1$ and $2 M(M=1, \ldots, N / 2)$ is

$$
|n\rangle_{M}=\sum_{n_{1}, n_{2}=1}^{d_{1}}\left(T_{M}^{2}\right)_{n_{1}, n_{2}}^{n}\left|n_{1}\right\rangle_{2 M-1} \otimes\left|n_{2}\right\rangle_{2 M},
$$

where $n=1, \ldots, d_{2}^{\mathrm{k}}$. In the $i$-th step, we can use the same formula to express the basis in $\mathcal{H}_{i}^{\mathrm{k}}$ in terms of $\mathcal{H}_{i-1}^{\mathrm{k}}$ just by replacing $T^{(2)}$ by $T^{(i)}$. The orthonormality of the basis gives us the condition

$$
\left.\left.\sum_{n_{1}, n_{2}=1}^{d_{i-1}} \overline{(} T_{M}^{(i)}\right)_{n_{1}, n_{2}}^{n^{\prime}} \overline{(} T_{M}^{(i)}\right)_{n_{1}, n_{2}}^{n}=\delta_{n^{\prime}, n}
$$

ie, $T$ must be an isometry. The final state can be easily written in terms of the $T^{(i)}$, but we will not do that here. Instead, we will use a graphical representation as we did in the case of MPS. 
(a)

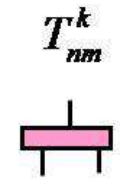

(b)

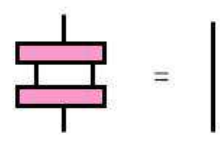

(c)

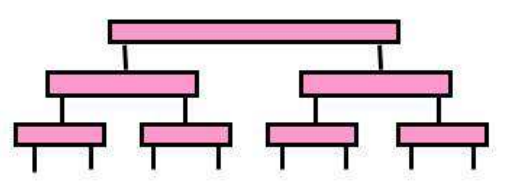

Figure 5. Tensor network representation of the TPS. (a) Each tensor $T$ is represented by a square with three indices. (b) the fact that $T$ is an isometry can be represented as a line when we contract $T$ and $\bar{T}$. (c) A TTS.

Let us start out representing the tensors $T$ as in Fig. 5(a). The orthonormality condition (45) can be thus graphically illustrated as in Fig. 5(b). That is, contracting the indices $n, m$ of the tensor with its complex conjugate gives a delta function (here represented by a line). Any state $|\Psi\rangle$ obtained with this renormalization scheme will have the structure of Fig. 5(c). That is, it will consist of different isometries $T$, characterizing the truncation of the Hilbert space of pairs of subsystems, which are contracted according to the diagram [53]. Note that we could have joined more than two spins in the first step, or in successive steps, in which case we would have obtained a similar diagram but in which the tensors would have more indices. One calls this class of states Tree Tensor States (TTS) since the diagram resembles a tree.

By looking at the tree structure of a state (Fig. 5(c)) it is very easy to notice that the states may violate the area law. In fact, if we look at a block of contiguous spins, we can deform the diagram and see how many links connect that block with the rest. Depending on where we take the block, the number may vary. In the figure, for instance, if we take a block with spins $2 M-1$ and $2 M$, then they will be connected by a single bond to the rest of the spins. If we take instead the spins 2 and 3 , they are connected by two bonds to the rest. It is easy to realize, as in the case of MPS, that the entropy of the block is bounded by the sum of the logarithms of the dimension of the bonds that 
connect the block with the rest. For some block, this entropy is bounded by a constant, whereas for some other ones it is bounded by $c \log L$, where $L$ is the number of spins in the block. Thus, a TTS violates the area law, although only mildly. In fact, given that critical systems typically have a logarithmic correction to the area law [36], it is natural to try to describe them with TTS, and thus with the renormalization group procedure exposed here, as it is usually done.

\subsection{Expectation values}

Expectation values of observables in a TTS can be easily evaluated, as it was the case for MPS. Let us take, for example, an observable acting on a give site, ie, $\langle\Psi|\sigma| \Psi\rangle$. We can write this expectation value as a contraction of two TTS, $\Psi$ and $\bar{\Psi}$, in which the operator $\sigma$ is sandwiched in between (Fig. 6(a)). Using the fact that all $T$ 's are isometries (ie Fig. 5(b)), we can heavily simplify the structure of this contraction. For example, in the figure the tensors on the right which are in a dimmer color can be substituted by straight lines, so that we obtain the tensor contraction of Fig. 6(b). Now, by redrawing it we obtain that of Fig. 6(c), which can in turn be written as a product of matrices, as it was the case with MPS. When we have a product of local observable, we can follow the same procedure. Just by having a look at the diagram, we can eliminate some of the tensors and replace them by lines, obtaining at the end a simple structure which can be easily contracted.

\subsection{Renormalization group}

As before, we can build a specific way of performing the renormalization, which is nothing but the standard one (but in position space instead of momentum or energy space). As in the case of the real-space renormalization group reviewed in previous sections, the simplest method consist of trying to minimize the energy every time we perform a renormalization step.

Let us consider a Hamiltonian of the form (16) (we could take other Hamiltonians with longer range interactions, but for illustration purposes we take the simplest one). First, we take $h_{1}$, acting on spins 1 and 2 , and determine the subspace of dimension $d_{2}^{\mathrm{k}}$ with lowest energy. That is, we diagonalize $h_{1}$ and take the subspace spanned by the $d_{2}^{\mathrm{k}}$ lowest eigenvalues. The projector on that subspace defined the isometry $T_{1}^{(2)}$. We do the same with $h_{3}, h_{5}$, etc. Then, we project the whole Hamiltonian onto the subspace (which is build as a tensor product of the selected ones), obtaining a new Hamiltonian with nearest neighbor interactions only. The reason is that the projection of $h_{2 M-1}$ is supported on the subspace of the new particle $M$, whereas $h_{2 M}$ is on that of particle $M$ and $M+1$ only. If we continue in this vein, we will obtain a renormalized Hamiltonian at each step. The ground state of the final Hamiltonian will thus have the structure of a TTS. Furthermore, this procedure may converge to a particular Hamiltonian, at least if we consider translationally invariant systems, and which is the fixed point operator of the renormalization group flow. This is the one which one 
(a)

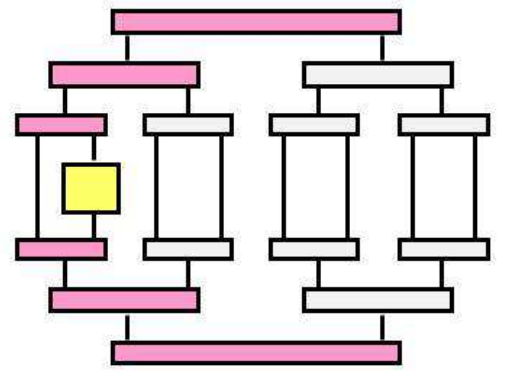

)

\section{$\langle\Psi|\sigma| \Psi\rangle$}

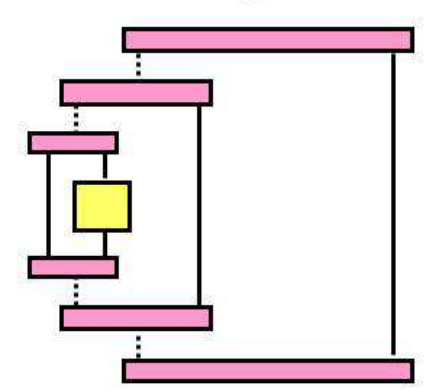

(c)

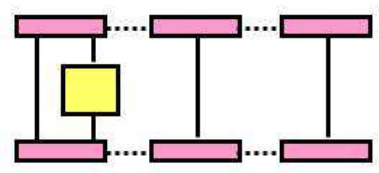

(b)

Figure 6. Expectation values of observables in a TTS. (a) Contraction of the TTS with an observable and its conjugate. (b) Using the fact that the $T$ 's are isometries we can get rid of several boxes (tensors); (c) reordering the indices we obtain a product of matrices.

usually analyzes in renormalization group to determine the possible phases depending on the different constants parametrizing $H$.

Of course, one may go beyond this procedure and try to minimize the energy directly as a function of all the tensors appearing in the tree, in much the same way one does in DMRG [79, 80, 81, 82. In fact, one can use similar techniques to those introduced in that context in order to perform this task in an efficient way. For instance, one may fix all the T's except for one, and minimize the energy with respect to that one. Then proceed with the next, and so on, until all the tensors (and thus the energy) converge. One can also choose all the tensors in a row equal, in order to emulate translationally invariance (this will occur anyway with the renormalization procedure given above for Hamiltonians with that symmetry), at the expense that one has to minimize with respect to all the tensors in one row at the same time. Furthermore, one may look for quasi-scale invariant solutions, in which all the tensors are chosen to be the same. Note, however, that unlike the case of MPS the state is not translationally invariant, since the spins are treated on different footing. That is, two spins that lie next to each other may not be grouped until the final step of the renormalization procedure. This indicates that this method may give good qualitative results (in most of the times, enough to determine the phases appearing in the problem), although not as precise as in DMRG. 


\subsection{Matrix Product States vs Tree Tensor States}

Given a MPS it is relatively simple to express it as a TTS. One way of seeing that is by considering the sequential generation of MPS explained in previous sections. There, we saw that a MPS of bond dimension $D$ can be generated by using a sequence of unitary operators acting on $\log D+1$ spins (see Figs. 4, 7). If we group $\log D$ spins into a single one, so that the new chain has $N / \log D$ big spins, those unitary operations become between nearest neighbors only. Now, let us consider the last of such spins. Obviously, there exists a unitary operation acting on it and the previous one that disentangles it from the whole chain (such unitary is the inverse of the one we apply in the sequential operation). The same applies to any big spin, since we can consider it as the last one of a sequentially generated state (we could start from the next one, go around the rest of spins, and end up in that particular one). This implies that we can, for example, act on every second big spin of the chain and its neighbor to the left with a unitary operation and disentangle them completely from the rest (see Fig. 7). The remaining state of the rest of the spins will still be a MPS with bond dimension $D$, so that we can apply exactly the same procedure. By iterating, we can completely disentangle all the big spins. The procedure we have carried out is nothing else but the one described at the beginning of this section, but with the big spins. This means that the state of the

big spins can be written as a TTS, and thus the original one too. The opposite is also true. Given a TTS we can always use the procedure described in previous sections to write it as a MPS. As discussed above in the context of the area law, the maximal rank of the density operators $\rho_{M}$ will be $O(\log N)$, and thus this determines the largest bond dimension, $D$, of the MPS.

\subsection{Other remarks}

It is very clear that one can apply the same techniques described in the previous section for MPS to TTS. For example, one can minimize the energy, determine the best approximations, time evolution, etc, by getting the tensors $T$ of the TTS variationally, going one by one (while fixing the rest), as in DMRG. The only point where one has to be careful is that, in this case, the fact that each $T$ must be an isometry plays an important role. In principle, the minimization of the energy, etc, for each tensor should take this constraint into account. However, as it was in the case of MPS, one can 'pull' this constraint so that in practice it is irrelevant. The idea here is to perform the minimization in some particular order. Let us take a position in the first row, fix the rest, and find the optimal tensor at that position (without imposing the isometry condition). Then, we determine a singular value decomposition of the tensor between the upper index and the other two, keeping at the end only the isometry. That is, we pull the non-isometric part upwards, and include it in the next tensor. Thus, at the next step, we minimize with respect of the tensor above the one before and continue in the same way until we reach the uppermost position. Then, we go again to the first row and repeat the whole procedure until we converge. Apart from that, we can also define 


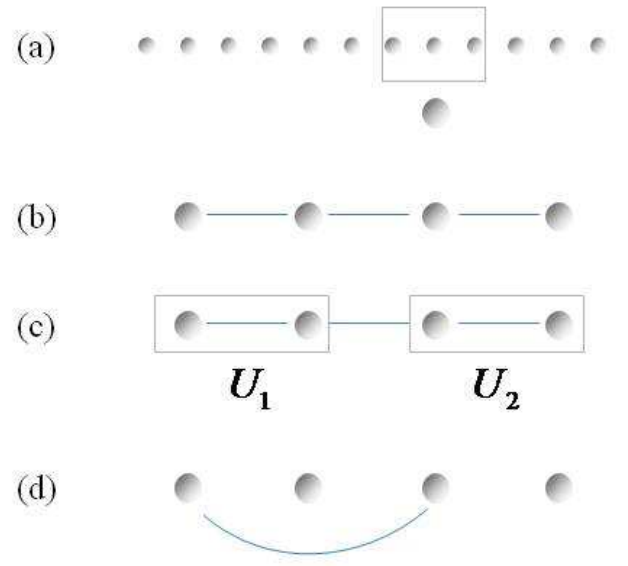

Figure 7. A MPS can be expressed as a TTS. (a) We group $\log D$ spins to build bigger spins, (b), which are entangled and form an MPS of bond dimension $D$. (c) Unitary operators acting on pairs of spins disentangle half of them, and leave the rest in a MPS of bond dimension $D$. We can continue this procedure until we have a single spin.

tree operators in analogy with matrix product operators, and thus carry out calculations at finite temperature or with mixed states.

It is interesting to view what happens if we apply the renormalization group procedure reviewed in this section to a finitely correlated state. In that case, one can solve the problem exactly and classify the fixed points of that procedure, obtaining the states that survive it [114.

\section{Multiscale Entanglement Renormalization Ansatz}

A more sophisticated way of implementing a real-space renormalization group was introduced by Ma and Dasgupta [83, and later successfully used by Fisher [84, 85] in the context of random quantum spin systems. Their blocking scheme is peculiar in the sense that one does not block several spins into one superspin as described before, but maps $n$ spins into $n^{\prime}<n$ spins, in such a way that locality in the interactions is preserved. This constraint is of crucial importance as it turns out that this is precisely the extra ingredient needed to extend those block-transformation to higher dimensions such that an area law for the entanglement entropy can be obtained. The idea of 
mapping $n$ spins into $n^{\prime}<n$ spins is also the basis of the multi-scale entanglement renormalization procedure introduced independently by Vidal [54, 55].

To sketch this approach, let us consider a quantum spin chain with only nearest neighbour interactions. A typical blocking scheme of Ma-Dasgupta-Fisher in the case of Heisenberg interactions would map 4 nearest neighbor spin $1 / 2$ 's $(k, k+1, k+2, k+3)$ into 2 new spin $1 / 2$ 's $\left(k^{\prime}, k^{\prime}+1\right)$. This is done in such a way that the renormalized Hamiltonian still only exhibits nearest neighbour interactions. In other words, the isometry $U_{k^{\prime}, k^{\prime}+1 ; k, k+1, k+2, k+3}$ used in the RG step has the following effect on the Hamiltonian $H=\sum_{k} h_{k, k+1}$ :

$$
\begin{aligned}
& U\left(h_{k-1, k}+h_{k, k+1}+h_{k+1, k+2}+h_{k+2, k+3}+h_{k+3, k+4}\right) U^{\dagger} \\
& =\bar{h}_{k-1, k^{\prime}}+\tilde{h}_{k^{\prime}, k^{\prime}+1}+\bar{h}_{k^{\prime}+1, k+4} .
\end{aligned}
$$

Such RG steps can again be implemented recursively, and this has been done with very big success for random antiferromagnetic spin chains: the isometries $U$ are found using standard second order perturbation theory, and the blocking becomes more accurate as a function of the blocking step. In a similar vein, this method has been used to simulate Ising spin systems with random ferromagnetic interactions and random transverse fields [84, 85], in which case 4 spins are mapped to 3 spins in such a way that the renormalized Hamiltonian only exhibits nearest neighbour interactions.

In analogy with the TTS, the quantum states that are generated during such a blocking scheme can easily be represented using isometries (see Fig. 8). One of the most interesting features of the class of states generated during such a $R G$ procedure is the fact that they can be critical and scale-invariant. Indeed, the Dasgupta-Ma-Fisher real-space RG method has been used to extract critical exponents. By looking at the structure of isometries, one indeed observes that the Schmidt rank when cutting the chain in two halves can grow as the logarithm of the size of the chain, as in the TTN: if one considers a periodic arrangement of the isometries as in Fig. 8, a logarithmic number of layers of blocking steps can contribute to generating entanglement. This has to be contrasted to the case of MPS, where the Schmidt number with respect to any cut is always bounded by a constant. Another very important property of the states obtained like that is that one can efficiently calculate expectation values of local operators: using the RG-scheme, one can represent local operators in the effective basis generated after consecutive RG-steps, and due to the exponential shrinking of the number of spins at every step, local operators will always remain local, up to the last level of the tree where any expectation value can trivially be calculated. More precisely, local operators will never act on more than a constant number of renormalized spins during the renormalization flow. This can be proven as follows: given an operator acting on $x_{k}$ nearest neighbor spins after $k$ iterations, then there exist constants $0<c_{1}<1$ and $c_{2}>1$ that depend on the RG blocking such that the range of the operator at the next level is bounded by $x_{k+1} \leq c_{1}\left(x_{k}+c_{2}\right)$. One can easily check that $x_{n}$ is always bounded above by $\max \left(c 1 . c 2 /(1-c 1), x_{0}\right)$ wich is a constant independent of the number of spins. In the case of the $4 \rightarrow 2$ isometry, $c_{1}=1 / 2, c_{2}=6$ and hence the bound is 
(a)

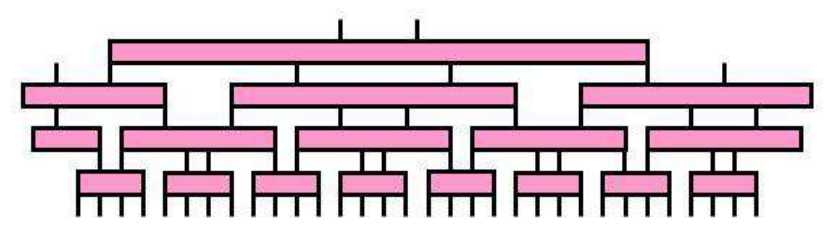

(b)

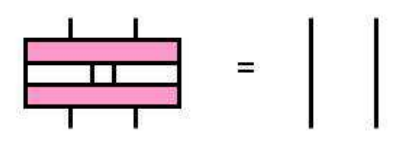

(c)

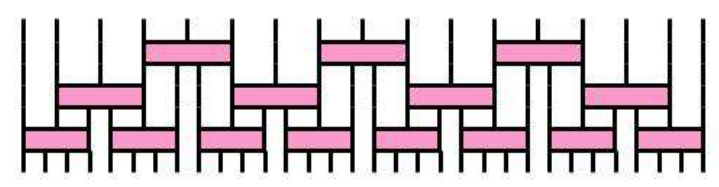

Figure 8. (a) Translational invariant blocking scheme a la Ma-Dasgupta-Fisher mapping 4 spins to 2 by isometries. (b) Every building block in the scheme consists of isometries. (c) Alternative blocking scheme as e.g. used for the Ising model mapping 4 spins to 3 .

$\max \left(6, x_{0}\right)$; for a general $n \rightarrow m$ scheme, $c_{1}=m / n, c_{2}=2 .(n-1)$.

Obviously, this class of states encompasses the class of TTS, as the latter is obtained in the special case of $n \rightarrow 1$ blocking schemes. However, this new class of states also shares the lack of translational symmetry with the TTS, as opposed to the case of MPS.

Now, a crucial step can be made in order to obtain a much more powerful method. One can choose the isometries at will, something which can lead to very different isometries than those obtained by second order perturbation theory. The resulting renormalization scheme is precisely the one introduced by G. Vidal and the underlying states were called multiscale entanglement renormalization ansatz (MERA) [54, 55]. This class of states was introduced by G. Vidal, and his construction was inspired by ideas originating in the field of quantum information theory of how to parameterize states using quantum circuits. He also proposed to obtain the isometries variationally. In a typical realization of a MERA, the states are parameterized by specifying a periodic pattern of isometries (the free parameters are exactly the isometries) as shown in Fig 9 and, furthermore, the isometries are themselves decomposed into a sequence of so-called disentangling unitaries and isometries (see Fig 9(b)). Apart from historical reasons, the incentive for splitting the isometries into smaller building blocks is that this allows 
(a)

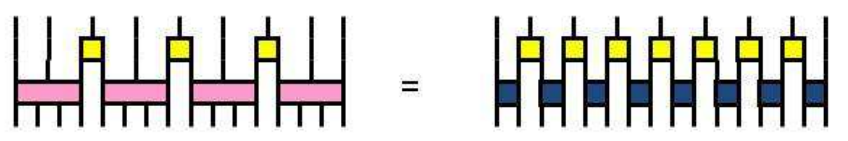

(b)

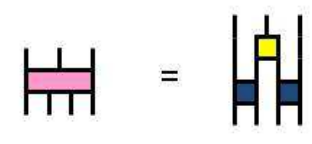

(c)

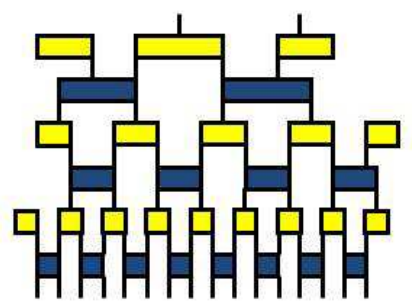

Figure 9. Multiscale Entanglement Renormalization Ansatz: (a),(b) Decomposing the isometries in the Ma-Dasgupta-Fisher scheme into a sequence of disentangling unitaries and isometries; (c) Typical representation of a MERA as a sequence of unitaries and isometries.

for a more efficient calculation of local expectation values; however, the disentangling unitaries are already implicitly present in the Ma-Dasgupta-Fisher blocking scheme. There is however also a very nice intuitive interpretation of the effect of those unitaries as so-called disentanglers: before doing a blocking, those disentanglers take care of removing entanglement within the block with the outside.

Due to the fact that MPS can be represented as TTS and TTS are a special case of MERA, it is clear that the class of MERA encompasses the class of MPS. As already explained in the context of the Ma-Dasgupta-Fisher RG-scheme, expectation values of local observables can easily be calculated by doing consecutive coarse-graining steps on the obervable of the form $\hat{O} \rightarrow U^{\dagger} O \hat{\otimes} \mathbf{1} U$. Due to the exponential shrinking of the number of spins, it is guaranteed that the renormalized observables $\hat{O}$ remain local at all steps.

In analogy to MPS and TTS, a variatonal calculation can now be done as an alternating optimization over the degrees of freedom in the state [86]. In the case of MERA, those degrees of freedom are the isometries, but unlike the case of MPS and TTN, those optimizations can not be mapped to alternating least squares problems; instead, a direct optimization over isometries has to be done, which is a nonlinear optimization problem that is more difficult and subtle to control due to the occurrence 
of local minima. Nevertheless, Vidal and collaborators have obtained impressive results, and e.g. calculated critical exponents of quantum spin chains to a very good precision by imposing a scale-invariant structure of the MERA [87, 88]. Such scale-invariant MERA have also been studied from the point of view of real-space RG transformations [91] and of quantum channels and quantum information theory [89, 90]. A further development is the formulation of a real- and imaginary-time evolution with MERA [92].

\section{Higher dimensions}

In principle, one can use all the previous constructions in higher dimensions. For instance, a MPS may approximate a $2 \mathrm{D}$ system, if we view it as a spin chain (ie we place the spins one after each other) [18, 19, 20]. However, the validity of the methods explained in previous sections will be questionable as soon as the system becomes sufficiently big such that it cannot longer be viewed as a 1D one. A way of expressing mathematically this intuition is through the area law. One expects that the entropy of a region increases with the number of spins at the border. For a MPS, it is simple to see that there will be regions for which the the entropy will not scale at all in that way. With TTS the same thing happens: whereas for some regions the entropy will grow even as the logarithm of the number of spins on that regions, for some other regions it will not grow at all. Apart from that, they will not give a translationally invariant state, as it should be for homogeneous problems. Nevertheless, the renormalization scheme that originates the TTS may be still applied to higher dimensional systems giving reasonable results for sufficiently small systems [93]. The MERA can be chosen to fulfill the area law, and thus they may be more appropriate than MPS and TTS for 2 and higher spatial dimensions. The construction can be immediately adapted from the $1 \mathrm{D}$ one. The issue of translational invariance still remains and thus the result may depend on how the tensors are chosen. Besides that, the minimization with respect to the tensors composing the TPS cannot be carried out as efficiently as with TTN, since it becomes difficult to avoid imposing the unitarity (or isometry) condition on the tensors. In any case, the first results on a $2 \mathrm{D}$ frustrated system in a $2 \mathrm{D}$ lattice have been recently reported, reveling a great potentiality of the method 94 .

Here we will mostly consider PEPS, which do not suffer from some of those drawbacks, and for which it has been explicitly proven to efficiently approximate a large set of problems, as mentioned in the introduction. The prize one has to pay is that the determination of expectation values has to be carried out approximately, as opposed to what happens with MPS, TTS and MERA. In practice, this does not pose a crucial problem since the error in the approximation can be easily estimated and made arbitrarily small by increasing the computational resources. PEPS algorithms have been recently applied a variety of $2 \mathrm{D}$ problems with very promising results both for finite [44, 95, 96, 97] and infinite [45, 98, 99] systems. We will also briefly mention other states at the end of the section. 


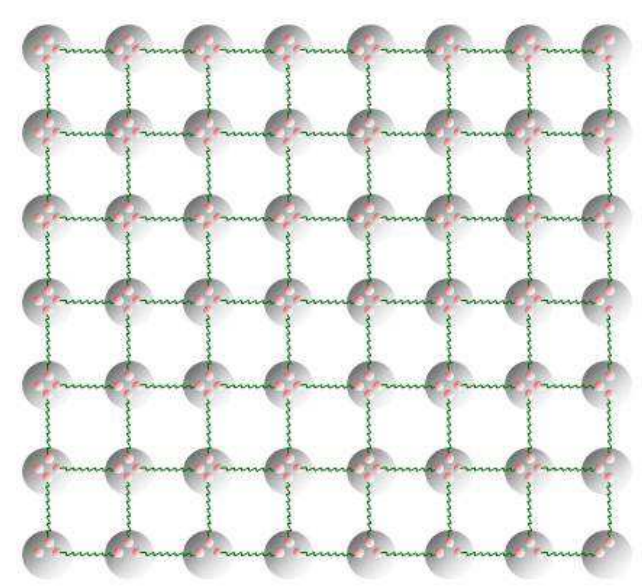

Figure 10. Projected entangled pair state (PEPS). As in the 1D case, each spin is replaced by four ancillas, which are maximally entangled with their neighbors. The state is produced by locally mapping the states of the ancillas onto the original spins.

\subsection{Projeted Entangled Pair States in 2 dimensions}

We extend here the construction of MPS from a previous section to 2D [44]. For that, we consider $N$ spins in a square lattice. We replace every spin by four auxiliary ones (Fig. 10), each of them in a maximally entangled state of dimension $D$ with a nearest neighbor (except for the borders). We then define a map, $P_{M}$, acting on each of the sites, $M$, that transforms the state of the auxiliary spins into the one of the original spins. Now, we can write the map at site $M$ in a particular basis, so that

$$
P_{M}=\sum_{\alpha, \beta, \gamma, \delta=1}^{D} \sum_{n=1}^{d_{1}}\left(A_{M}^{n}\right)_{\alpha, \beta, \gamma, \delta}|n\rangle(\langle\alpha, \beta, \gamma, \delta|,
$$

where we have used the same notation as before, but now we have fixed the dimension $D$ to simplify the notation. The state obtained after this procedure is called a PEPS. We can write the PEPS in the spin bases, in which case we will have that the corresponding coefficients will be given by the contraction of the tensors $A$ according to the auxiliary indices (Greek letters). This is represented in Fig. 11.

Given the PEPS construction, it is very simple to understand that they satisfy the area law. First, if we look at the state of the auxiliary particles, the entropy of a region $A$ will be equal to the number of cuts, $n_{A}$, of the entangled pairs across the border of 
(a)

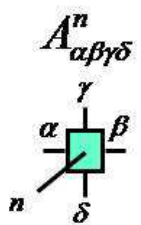

(b)

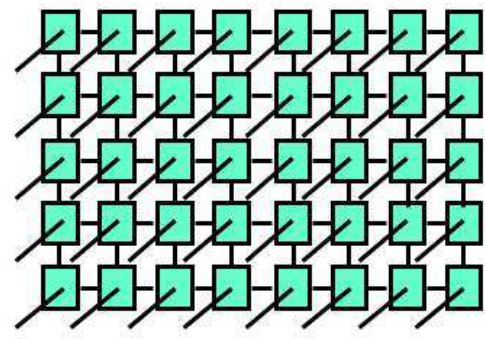

Figure 11. Tensor network representation of a PEPS. (a) Representation of the tensor corresponding to a single site. The indices in the plane correspond to the auxiliary particles, whereas the one orthogonal is the spin one; (b) Representation of the whole state where the auxiliary indices are contracted.

the region times $\log D$. In fact, the rank of the reduced density operator will be exactly $D^{n_{A}}$. On the other hand, the maps $P$ cannot increase the rank of the density operator, and thus we obtain the area law for the real spins, given that the entropy of an operator is upper bounded by the logarithm of its rank.

The expectation values of observables in a PEPS have a similar structure to those in a MPS (see Fig. [12(a)). We have to sandwich the operator between the tensors corresponding to $\Psi$ and $\bar{\Psi}$ as shown in the figure. At the end, everything boils down to contracting a tensor of the form shown in Fig. 12(b). This is very hard, in general. The reason is that if we start contracting the tensors appearing there, the indices will proliferate and in the middle of the calculation we will have of the order of $\sqrt{N}$ indices, which amounts to having an exponential number of coefficients. This is very different to what occurs in $1 \mathrm{D}$, in which chase the linear geometry makes it possible to contract the tensors while always keeping two indices at most.

One way to proceed is to realize that the tensor network displayed in Fig. 12(b) can be viewed as follows. The first row can be considered as a tensor which in turns is built out of smaller tensors, in much the same way as a MPS is built out of the tensors $A$. The next row can be viewed as a MPO. Thus the contraction of the first row with 
the second will give rise to a tensor like the first one but with a higher bond dimension (the square of the original one). If we contract the next row, we will multiply the bond dimension again, and at some point it will be impossible to proceed. Instead, what we can do is to try to approximate the first plus second row by a tensor of the form of a MPS but with reduced dimensions (Fig. 12(c)). For that, we use the technique explained in previous sections on how to approximate states by MPS optimally. We can now iterate this technique by adding the third row, and again decreasing the bond dimension of the resulting tensor (Fig. 12(d)). In this way, we can keep all the dimensions under control and obtain the desired expectation value. A word of caution must be called for here. There is no guarantee that we will be able to obtain a good approximation at the end if we decrease the dimensions of the tensors. In practice, however, we have performed all those approximations and obtained very good results. Furthermore, one is always aware of the error made in the contraction, since it can be determined through the procedure itself. The reason why one obtains very accurate results in practice can be qualitatively understood as follows. The contraction we are performing can be viewed as evaluating a kind of partition function of a 1D quantum system at non-zero temperature. In fact, the MPO of each row can be interpreted as a transfer operator, in much the same way as in 1D quantum (or 2D classical) systems. If that matrix has a gap, which occurs outside the critical points, the procedure we are carrying out will tend to give the eigenvector of the transfer matrix corresponding to the maximal eigenvalue. If this eigenvector has an efficient representation as a MPS, then our procedure will succeed. Even though there is no proof for that, the problem of finding the maximal eigenvector of the transfer matrix in $1 \mathrm{D}$ is very reminiscent of that of finding the ground state of a Hamiltonian, for which a MPS provides a good approximation (for short range interactions, although numerically it also works for longer range interactions).

Once one has an efficient algorithm to determine expectation values, one can literally translate all the algorithms developed for MPS to PEPS. In particular, one can approximate time evolutions, thermal states, etc, with this methods. In Ref. [71] those algorithms are explained in great detail.

Let us now explain why PEPS are well suited to describe spins in thermal equilibrium in the case of local Hamiltonians in any dimension. Let us write $H=\sum h_{\lambda}$, where $h_{\lambda}$. For simplicity, we will assume that each $h_{\lambda}$ acts on two neighboring spins although this can be generalized for $h_{\lambda}$ acting on a small region. We first rewrite the (unnormalized) density operator $e^{-\beta H}=\operatorname{tr}_{B}[|\Psi\rangle\langle\Psi|]$, where $|\Psi\rangle=e^{-\beta H / 2} \otimes|\Phi\rangle_{A B}$ is a purification [47] and $|\Phi\rangle_{A B}$ a pairwise maximally entangled state of each spin with another one, the latter playing the role of an environment. We will show now that $|\Psi\rangle$ can be expressed as a PEPS. We consider first the simplest case where $\left[h_{\lambda}, h_{\lambda^{\prime}}\right]=0$, so that $|\Psi\rangle=\prod_{\lambda} e^{-\beta h_{\lambda} / 2} \otimes \mathbf{1}|\Phi\rangle_{A B}$. The action of each of the terms $e^{-\beta h_{\lambda} / 2}$ on two spins in neighboring nodes can be viewed as follows [100, 43]: we first include two auxiliary spins, one in each node, in a maximally entangled state, and then we apply a local map in each of the nodes which involves the real spin and the auxiliary spin, that ends up in $|0\rangle$. By proceeding in the same way for each term $e^{-\beta h_{\lambda} / 2}$, we end up 


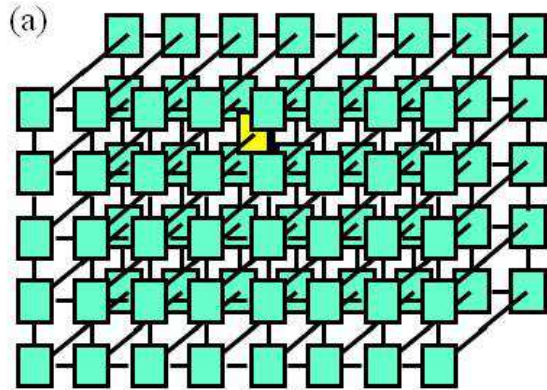

(c)
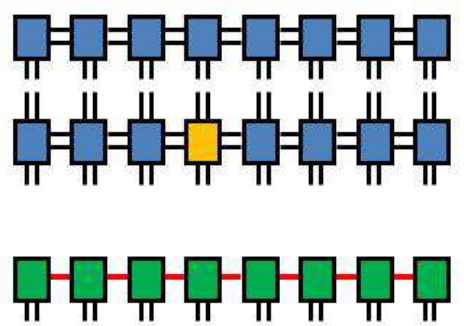

(b)

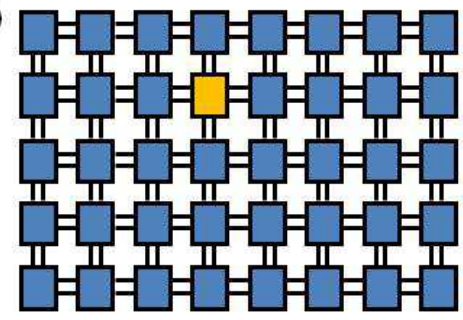

(d)

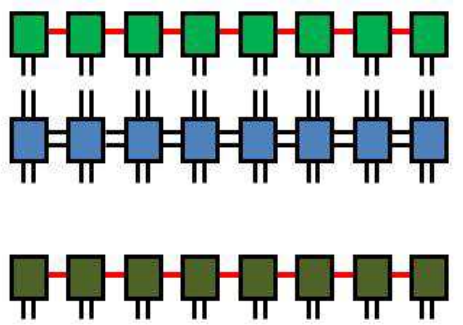

Figure 12. Determination of expectation values with PEPS. (a) The tensor network corresponding to the expectation value is obtained by sandwiching the observable (here at the position 2,4 ) with $\Psi$ and $\bar{\Psi}$. (b) We can join pair of indices in each bond to have a single one. (c) In order to contract the resulting tensor, we observe that the first row has the same tensor structure as a MPS. Then, we contract the first row with the second, and approximate the result optimally by a set of tensors with the structure of a MPS. (d) We do the same thing with the next row, and continue in the same way until we find the result.

with the PEPS description. This is valid for all values of $\beta$, in particular for $\beta \rightarrow \infty$, i.e., for the ground state. In case the local Hamiltonians do not commute, a more sophisticated proof is required [51]. One can, however, understand qualitatively why the construction remains to be valid by using a Trotter decomposition to approximate $e^{-\beta H} \approx \prod_{m=1}^{M} \prod_{\lambda} e^{-\beta h_{\lambda} / 2 M}$ with $M \ll 1$. Again, this allows for a direct implementation of each $\exp \left[-\beta h_{\lambda} / 2 M\right]$ using one entangled bond, yielding $M$ bonds for each vertex of the lattice. Since, however, the entanglement induced by each $\exp \left[-\beta h_{\lambda} / 2 M\right]$ is very small, each of these bonds will only need to be weakly entangled, and the $M$ bonds can thus be well approximated by a maximally entangled state of low dimension. Note that the spins belonging to the purification do not play any special role in this construction.

\subsection{Other approaches}

For translationally invariant Hamiltonians, one may directly consider the limit $N \rightarrow \infty$. In that case, the PEPS is taken with identical maps $P$ (equivalently, tensors $A$ ), in as 
much the same way as MPS are chosen to be FCS. The states so constructed have been called iPEPS [45] and coincide with the vertex-matrix product ansatz introduced earlier [22. In that paper, another family of translationally invariant states was introduced, motivated by the interaction round the face models in statistical mechanics. In a square lattice, the tensors $A$ are at the vertices of the $\mathrm{B}$ sublattice. The physical index, $n$, of the tensor $A_{\alpha_{11}, \alpha_{12}, \alpha_{21}, \alpha_{22}}^{n}$ are associated to the vertices of the $\mathrm{B}$ sublattice, whereas the auxiliary indices, $\alpha_{i j}$, are at the bonds. Thus, the contraction of the indices in that case is different, since each auxiliary variable is common to four tensors (and not two, like in the other case, see Fig. 13) (see also [102, 103]. One can extend this last class of states to include other tensor structures as shown in Fig. 13(c). In that state, there are two kinds of tensors: those that are represented by circles and have one physical index and four auxiliary ones, and those represented by squares with no physical index [101. The reason for the construction of those tensors is that this is an effective way of increasing the entanglement among nodes without increasing the bound dimension, but just the number of tensors. Since the PEPS algorithms typically have a much milder dependence of the computational time with the number of tensors than with the bond dimension, this has a very positive effect on the algorithms. Finally, there is yet another interesting class of states introduced by Nishino and collaborators, who also extend the ones proposed by Sierra and Martin-Delgado as interaction round a face type. They naturally appear in the transfer matrix of 3D classical models. In those states, each tensor now belongs to a plaquette, and depends on all the physical and

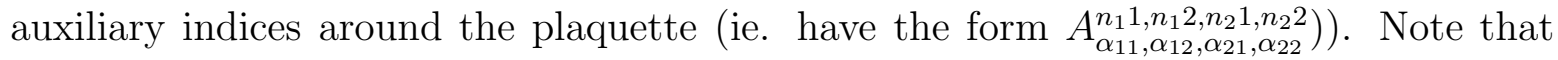
dropping the dependence on $n_{12}, n_{21}$ and $n_{22}$ on that tensor we obtain the one of Fig. 13(b).

We finally briefly mention other states which have an interesting property: stringbond-states [104] and the entangled-plaquette-states [105]. The coefficients of those states in the spin basis are expressed as products of other coefficients. In the first case, the latter coefficients are just MPS along different strings going through some spins in the lattice; in the second, they correspond to large overlapping plaquettes. The main feature of those states is that the expectation values can be calculated using Monte Carlo methods. Other interesting states displaying that property have been introduced by Sandvik [106, 107, 108].

\section{Summary and perspectives}

It is very remarkable that one can identify the corner of Hilbert space which is relevant to describe the ground and thermal equilibrium states for a large variety of Hamiltonians. Those states are the MPS and their generalization to higher spatial dimensions, the so-called PEPS. Here we have reviewed those and other families of states (including the TTS and MERA) which are a subclass of TPS. We have shown how most of them (all except PEPS) follow immediately from some real-space renormalization procedure. We have characterized them, and shown how one can build algorithms with them to perform 
(a)

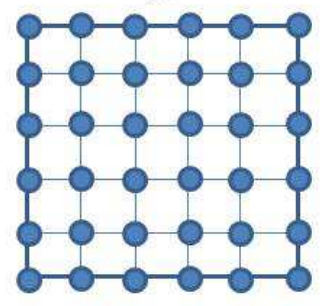

(b)

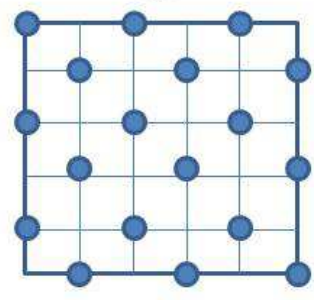

(c)

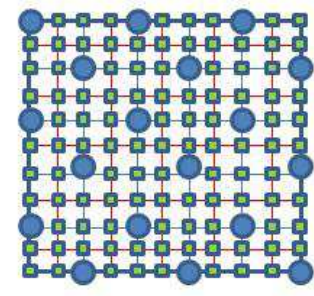

Figure 13. Representation of a vertex (a) and a face (b) matrix product ansatz. The tensors are specified by circles, and the auxiliary indices by the lines joining them. As one can see, each auxiliary index appears in two (a) and four (b) tensors, respectively.

different tasks, ranging from finding ground states, thermal states, evolutions, etc.

There remain very important problems in the context of TPS. First of all, it would be very interesting to prove beyond the intuitive arguments and the successful numerical results that the different procedures to determine expectation values of PEPS converge in practice. Also, even though we know that the state we are looking for is close to a MPS or a PEPS, nothing guarantees that our algorithm (DMRG or its extensions) will find it, although in all practical situations it does. Thus it would be very interesting to find conditions under which this will be the case. Note that there exist problems for which the ground state is a MPS but it cannot be found [109], or other in which it is a PEPS and it cannot be efficiently contracted, since that would violate the general believe in computer science that some problems are exponentially hard [110, 111]. Apart from that, when we have talked about the corner of Hilbert space we have always restricted ourselves to ground or thermal states of short-range interacting Hamiltonians. But, what happens for long-range Hamiltonians? Or, for time evolution? In the last case it can be shown that even in 1D a MPS can, in general, only approximate the state for short times [112, 113]. This indicates that the MPS are not well suited to describe time evolution for long times, and that the family of states describing that corner of Hilbert space is a completely different one. 
Another challenge is to find more efficient algorithms that work in higher dimensions. For example, the time resources associated to the algorithm based on PEPS to determine the ground state of a 2D Hamiltonian with open boundary conditions scales like $N^{2} D^{10}$, which allows to work with up to $20 \times 20$ lattices of spin $1 / 2$ particles with

$D=5$. For problems with periodic boundary conditions or higher dimensions, the dependence is even worse, which makes it unpractical. The same is true for the MERA algorithms in 2 and higher dimensions. In particular, in $2 \mathrm{D}$ it scales as $D^{16}[115]$. Thus, we have to find ways of determining the states more efficiently, or new families of states (related to PEPS) for which we can do this task much faster. One possibility which is currently exploited and is very promising consists of combining the TPS and other descriptions with Monte Carlo methods [104, 105, 106, 107, 108].

\section{Acknowledgments}

We wish to thank the people who has collaborated with us on the subject of this manuscript. Special thanks go also to: M. C. Banuls, J. von Delft, W. Duer, J. GarciaRipoll, M. Hastings, C. Kraus, J. I. Latorre, I. Mc Cullogh, M. A. Martin-Delgado, F. Mezzacapo, V. Murg, T. Nishino, R. Orus, B. Paredes, D. Perez-Garcia, B. Pirvu, D. Porras, E. Rico, M. Sanz, N. Schuch, U. Schollwöck, G. Vidal, S. White, and M. Wolf. This paper was written during a stay in the Aspen Center for Physics, participating in the quantum simulation program. We acknowledge financial support from the EU project QUEVADIS, the German Science Foundation (FOR635), the excellence cluster Munich Center for Advanced Photonics, and the FOQUS SFB from the Austrian Science Foundation.

\section{References}

[1] Wilson K G 1975 Rev. Mod. Phys. 47773

[2] Kadanoff L P 1966 Physics 2263

[3] White S R 1992 Phys. Rev. Lett. 692863

[4] White S R 1992 Phys. Rev. B 4810345

[5] Peschel I Wang X Kaulke M and Hallberg K 1998 Density-Matrix Renormalization Springer-Verlag

[6] Schollwöck U 2005 Rev. Mod. Phys. 77259

[7] Östlund S and Rommer S 1995 Phys. Rev. Lett. 753537

[8] Dukelsky J Martin-Delgado M A Nishino T and Sierra G 1998 Europhys. Lett. 43457

[9] Takasaki H Hikihara T and Nishino T 1999 J. Phys. Soc. Jpn 681537

[10] Verstraete F Porras D and Cirac J I 2004 Phys. Rev. Lett. 93227205

[11] Kramers H A and Wannier G H 1941 Phys. Rev. 60263

[12] Affleck I Kennedy T Lieb E H and Tasaki H 1987 Phys. Rev. Lett. 59799.

[13] Affleck I Kennedy T Lieb E H and Tasaki H 1988 Commun. Math. Phys. 115477

[14] Fannes M Nachtergaele B and Werner R F 1992 Comm. Math. Phys. 144443

[15] Klümper A Schadschneider A and Zittartz J 1993 Europhys. Lett. 24293

[16] Lange C Klümper A and Zittartz J 1994 Z. Phys. B 96267

[17] Perez-Garcia D Verstraete F Wolf M M and Cirac J I 2007 Quantum Inf. Comput. 7401

[18] Liang S and Pang H 1994 Phys. Rev. B 499214

[19] White S R 1996 Phys. Rev. Lett. 773633 
[20] Xiang T Lou J and Su Z 2001 Phys. Rev. B 64104414

[21] Nishino T and Okunishi K 1998 J. Phys. Soc. Jpn. 673066

[22] Sierra G and Martin-Delgado M A Preprint: cond-mat/9811170

[23] Niggemann H Klumper A and Zittartz J 1997 Z. Phys. B 104103

[24] Y. Hieida Y Okunishi K and Akutsu Y 1999 New J. Phys. 1

[25] Okunishi K and Nishino T 2000 Prog. Theor. Phys. 03541

[26] Nishino T Okunishi K Hieida Y Maeshima N and Akutsu Y 2004 Nucl. Phys. B 575504

[27] Nishino T Hieida Y Okunishi K Maeshima N and Akutsu Y 2001 Prog. Theor. Phys. 105409

[28] Maeshima N Hieida Y Akutsu Y Nishino T and Okunishi K 2001 Phys. Rev. E 64016705

[29] Gendiar A Maeshima N and Nishino T 2003 Prog. Theor. Phys. 110691

[30] Nishino Y Maeshima N Gendiar A and Nishino T Preprint arXiv:cond-mat/0401115

[31] Martin-Delgado M A Roncaglia M and Sierra G 2001 Phys. Rev. B 64075117.

[32] Srednicki M 1993 Phys. Rev. Lett. 71666

[33] Callan C G and Wilczek F 1994 Phys. Rev. B 33355

[34] Holzhey C Larsen F and Wilczek F 1994 Nucl. Phys. B 44424

[35] Vidal G Latorre J I Rico E and Kitaev A 2003 Phys. Rev. Lett 90227902

[36] Calabrese P and Cardy J 2004 J. of Stat. Mechanics: Theory and Experiment 0406 P002

[37] Eisert J Cramer M and Plenio M B Preprint arXiv:0808.3773

[38] Hastings M B 2007 J. Stat. Phys. P08024

[39] Wolf M M 2006 Phys. Rev. Lett. 96010404

[40] Gioev D and Klich I 2006 Phys. Rev. Lett. 96100503

[41] Wolf M M Verstraete F Hastings M B and Cirac J I 2008 Phys. Rev. Lett. 100070502

[42] Popp M Verstraete F Martin-Delgado M A Cirac J I 2005 Phys. Rev. A 71042306

[43] Verstraete F and Cirac J I 2004 Phys. Rev. A 70060302

[44] Verstraete F and Cirac J I Preprint arXiv:cond-mat/0407066

[45] Jordan J Orus R Vidal G Verstraete F and Cirac J I 2008 Phys. Rev. Lett. 1001250602

[46] Kraus, C V Schuch N Verstraete F and Cirac J I Preprint arXiv:0904.4667

[47] Verstraete F García-Ripoll J J and Cirac J I 2004 Phys. Rev. Lett. 93207204

[48] Zwolak M and Vidal G 2004 Phys. Rev. Lett. 93207205

[49] Verstraete F and Cirac J I 2006 Phys. Rev. B 73094423

[50] Osborne T J 2007 Phys. Rev. A 75042306

[51] Hastings M B 2007 Phys. Rev. B 76035114

[52] Hastings M B 2006 Phys. Rev. B $\mathbf{7 3} 085115$

[53] Fannes M Nachtergaele B and Werner, R F 1992 Journal of Statistical Physics 66939

[54] Vidal G 2007 Phys. Rev. Lett. 99220405

[55] Vidal G 2008 Phys. Rev. Lett. 101110501

[56] Levin M and Nave C P 2007 Phys. Rev. Lett. 99120601

[57] Gu Z C Levin M Wen X G 2008 Phys. Rev. B 78205116

[58] Wang L Kao Y J and Sandvik A W Preprint: arXiv:0901.0214

[59] Schöen C Solano E Verstraete F Cirac J I and Wolf M M 2005 Phys. Rev. Lett. 95110503

[60] Paredes B Verstraete F and Cirac J I 2005 Phys. Rev. Lett. 95140501

[61] Porras D Verstraete F and Cirac J I 2006 Phys. Rev. B 73014410

[62] Cazalilla M A and Marston J B 2002 Phys. Rev. Lett. 88256403

[63] Vidal G Efficient simulation of one-dimensional quantum many-body systems 2004 Phys. Rev. Lett. 93040502

[64] White S R and Feiguin A E 2004 Phys. Rev. Lett. 93076401

[65] Daley A J Kollath C Schollwöck U and Vidal G 2004 J. Stat. Mech.: Theor. Exp. P04005

[66] Schollwöck U and White S R Preprint cond-mat/0606018

[67] Sirker J and Klümper A 2005 Phys. Rev. B 71241101

[68] Hastings M B Preprint arXiv:0903.3253

[69] Bauls M C Hastings M B Verstraete F and Cirac J I Preprint arXiv:0904.1926 
[70] Murg V Verstraete F and Cirac J I 2005 Phys. Rev. Lett. 95057206

[71] Verstraete F Murg V and Cirac J I 2008 Advances In Physics 57143

[72] Perez-Garcia D Wolf M M Verstraete F and Cirac J I in preparation

[73] Weichselbaum A Verstraete F Schollwöck U Cirac J I and von Delft J Preprint arXiv:cond-mat/0504305v1

[74] Bennett C H Brassard G Crepeau C Jozsa R Peres A and Wootters W 1993 Phys. Rev. Lett. 70 1895

[75] Rommer S and Östlund S 1997 Phys. Rev. B 552164

[76] Vidal G 2007 Phys. Rev. Lett. 98070201

[77] Vidal G 2003 Phys. Rev. Lett. 91147902

[78] Korepin V and Verstraete F, In preparation

[79] Friedman B 1997 Journal of Physics: Condensed Matter 99021

[80] Martin-Delgado M A Rodriguez-Laguna J and Sierra G 2002 Physical Review B 65155116

[81] Nagaj D Farhi E Goldstone J Shor P and Sylvester I 2008 Physical Review B 77214431

[82] Shi Y Y Duan L M and Vidal G 2006 Phys. Rev. A $\mathbf{7 4} 022320$

[83] Ma S K Dasgupta C and Hu C K 1979 Phys. Rev. Lett. 431434

[84] Fisher D S 1994 Phys. Rev. B 503799

[85] Fisher D S 1995 Phys. Rev. B 516411

[86] Evenbly G and Vidal G 2009 Phys. Rev. B 79144108

[87] Pfeifer R N C Evenbly G and Vidal G Phys. Rev. A 79040301

[88] Montangero S Rizzi M Giovannetti V and Fazio R Preprint arXiv:0810.1414

[89] Giovannetti V Montangero S and Fazio R 2008 Phys. Rev. Lett. 101180503

[90] Giovannetti V Montangero S Rizzi M and R. Fazio R 2009 Phys. Rev. A 79052314

[91] Evenbly G and G. Vidal G Preprint: arXiv:0710.0692.

[92] Rizzi M Montangero S and Vidal G 2008 Phys. Rev. A 77052328

[93] Tagliacozzo L Evenbly G and Vidal G Preprint arXiv:0903.5017

[94] Evenbly G and Vidal G Preprint arXiv:0904.3383

[95] Isacsson A and Syljuasen O F 2006 Phys. Rev. E $\mathbf{7 4} 026701$

[96] Murg V Verstraete F and Cirac J I 2007 Phys. Rev. A 75033605

[97] Murg V Verstraete F and Cirac J I Preprint: arXiv:0901.2019

[98] Orus R and Vidal G Preprint: arXiv:0905.3225

[99] Bauer B Vidal G Troyer M Preprint arXiv:0905.4880

[100] Cirac J I Dr W Kraus B and Lewenstein M 2001 Phys. Rev. Lett. 86544

[101] Murg V Verstraete F and Cirac J I In preparation

[102] Hubener R Nebendahl V and Dur W, Preprint: arXiv:0904.1925

[103] Rico E Hubener R Montangero S Moran N Pirvu B Vala J and Briegel H J Preprint: arXiv:0811.1049

[104] Schuch N Wolf M M Verstraete F and Cirac J I 2008 Phys. Rev. Lett. 100040501

[105] Mezzacapo F Schuch N Boninsegni M and Cirac J I Preprint: arXiv:0905.3898

[106] Sandvik A W Preprint: arXiv:0710.3362

[107] Sandvik A W and Evertz H G Preprint: arXiv:0807.0682

[108] Sandvik A W and Vidal G Preprint: arXiv:0708.2232

[109] Schuch N Cirac J I and Verstraete F 2008 Phys. Rev. Lett. 100250501

[110] Verstraete F Wolf M M Perez-Garcia D and Cirac J I 2006 Phys. Rev. Lett. 96220601

[111] Schuch N Wolf M M Verstraete F and Cirac J I 2007 Phys. Rev. Lett. 98140506

[112] Schuch N Wolf M M Verstraete F and Cirac J I 2008 Phys. Rev. Lett. 100030504

[113] Calabrese P and John Cardy J 2007 J. Stat. Mech. P10004

[114] Verstraete F Cirac J I Latorre J I Rico E and M.M. Wolf M M 2005 Phys. Rev. Lett. 94140601

[115] Evenbly G Vidal G 2009 Phys. Rev. Lett. 102180406 